\title{
SLAMF1 regulation of chemotaxis and autophagy determines CLL patient response
}

\author{
Cinzia Bologna, ${ }^{1,2}$ Roberta Buonincontri, ${ }^{1,2}$ Sara Serra, ${ }^{2}$ Tiziana Vaisitti, ${ }^{1,2}$ Valentina Audrito, ${ }^{2}$ Davide Brusa, ${ }^{1,2}$ Andrea Pagnani, ${ }^{2,3}$ \\ Marta Coscia, ${ }^{4}$ Giovanni D'Arena, ${ }^{5}$ Elisabetta Mereu, ${ }^{6}$ Roberto Piva, ${ }^{6}$ Richard R. Furman, ${ }^{7}$ Davide Rossi, ${ }^{8}$ Gianluca Gaidano, ${ }^{8}$ \\ Cox Terhorst, ${ }^{9}$ and Silvia Deaglio ${ }^{1,2}$ \\ Department of Medical Sciences, University of Torino, Torino, Italy. ${ }^{2}$ Human Genetics Foundation (HuGeF), Torino, Italy. ${ }^{3}$ Department of Applied Science and Technology, Politecnic of Torino, Torino, Italy. \\ ${ }^{4}$ Division of Hematology, AO Città della Salute e della Scienza, Torino, Italy. ${ }^{5}$ Hematology and Stem Cell Transplantation Unit, IRCCS Centro di Riferimento Oncologico della Basilicata, Rionero in Vulture, Italy. \\ ${ }^{6}$ Molecular Biotechnology Center, Department of Molecular Biotechnology and Health Sciences, University of Torino, Torino, Italy. ${ }^{7}$ Weill Medical College of Cornell University, New York, New York, USA. \\ ${ }^{8}$ Division of Hematology, Department of Translational Medicine, "Amedeo Avogadro" University of Eastern Piedmont, Novara, Italy. "Department of Medicine, Division of Immunology, \\ Beth Israel Deaconess Medical Center, Harvard Medical School, Boston, Massachusetts, USA
}

\begin{abstract}
Chronic lymphocytic leukemia (CLL) is a variable disease; therefore, markers to identify aggressive forms are essential for patient management. Here, we have shown that expression of the costimulatory molecule and microbial sensor SLAMF1 (also known as CD150) is lost in a subset of patients with an aggressive CLL that associates with a shorter time to first treatment and reduced overall survival. SLAMF1 silencing in CLL-like Mec-1 cells, which constitutively express SLAMF1, modulated pathways related to cell migration, cytoskeletal organization, and intracellular vesicle formation and recirculation. SLAMF1 deficiency associated with increased expression of CXCR4, CD38, and CD44, thereby positively affecting chemotactic responses to CXCL12. SLAMF1 ligation with an agonistic monoclonal antibody increased ROS accumulation and induced phosphorylation of $\mathrm{p} 38$, JNK1/2, and BCL2, thereby promoting the autophagic flux. Beclin1 dissociated from BCL2 in response to SLAMF1 ligation, resulting in formation of the autophagy macrocomplex, which contains SLAMF1, beclin1, and the enzyme VPS34. Accordingly, SLAMF1-silenced cells or SLAMF1' primary CLL cells were resistant to autophagy-activating therapeutic agents, such as fludarabine and the BCL2 homology domain 3 mimetic ABT-737. Together, these results indicate that loss of SLAMF1 expression in CLL modulates genetic pathways that regulate chemotaxis and autophagy and that potentially affect drug responses, and suggest that these effects underlie unfavorable clinical outcome experienced by SLAMF $1^{10}$ patients.
\end{abstract}

\section{Introduction}

Chronic lymphocytic leukemia (CLL), the most common form of adult leukemia in Europe and North America, is characterized by the expansion of a population of mature B-lymphocytes that accumulate in the BM, lymphoid tissues, and the blood (1). Because of the highly variable course of the disease, molecular and genetic markers are important predictors of prognosis. Identification of aggressive CLL is currently based on the presence of genetic lesions, including deletions or mutations in the TP53, ATM, NOTCH1, and SF3B1 genes (2). Patients with aggressive CLL are also characterized by the absence of mutations in the immunoglobulin heavy chain V $(I G H V)$ genes and by the expression of CD38 and CD49d, among other markers (3-5). Instead, the only genetic abnormalities characteristic of CLL cases with a favorable prognosis are deletions at chromosome $13 q 14(6,7)$. This type of deletion involves microRNA-15 and -16, which target the antiapoptotic molecule BCL2 (8). Accordingly, the majority of CLL patients are characterized by a constitutively elevated expression of BCL2, suggesting that resistance to apoptosis plays an important role in the disease. This inference is supported by

Conflict of interest: The authors have declared that no conflict of interest exists. Submitted: June 11, 2015; Accepted: October 29, 2015.

Reference information: / Clin Invest. 2016;126(1):181-194. doi:10.1172/JCI83013. the clinical benefits obtained using drugs that target BCL2 in the management of these patients (9).

Autophagy is a membrane-trafficking mechanism that delivers cytoplasmic constituents into the lysosome for bulk degradation (10, 11). Basal autophagy is constitutively active at low levels in most tissues and maintains protein and organelle quality through the selective elimination of damaged intracellular material. The autophagy pathway is dramatically upregulated in response to stress and starvation, where it plays a critical role in removing damaged organelles and aggregated proteins, whose accumulation is toxic (12). Activation of autophagy relies on the formation of a molecular macrocomplex that contains the scaffold protein beclin1 (or ATG6) and the class III phosphatidyl inositol 3-kinase (PIK3C3 or VPS34), among other molecules. Under physiological conditions, the $\mathrm{BH} 3$ domain of beclin1 is bound to and inhibited by BCL2. This interaction can be disrupted by phosphorylation of BCL2, providing a molecular connection between autophagy and apoptosis (13). In cancer, autophagy plays dual roles, acting both as a tumor suppressor mechanism - by preventing accumulation of damaged proteins and organelles and as a prosurvival mechanism, promoting tumor growth $(14,15)$. In addition, recent findings obtained in patients with diffuse large B cell lymphoma indicate that low expression of beclin1 is associated to resistance to conventional chemotherapy regimens (16), suggesting that failed activation of autophagy may condition therapeutic 
Table 1. TFS and OS of CLL patients categorized on the basis of SLAMF1 expression

\begin{tabular}{ccccccccc} 
& \multicolumn{9}{c}{ TFS } & \multicolumn{5}{c}{ OS } \\
& Events & Total & Median & $95 \%$ Cl & Events & Total & 10 years & $95 \%$ Cl \\
SLAMF1 $>6 \%$ & 92 & 209 & $7.6 \mathrm{yr}$ & $5.3-9.8$ & 11 & 233 & $94.7 \%$ & $91.4-98.6$ \\
SLAMF1 $\leq 6 \%$ & 30 & 45 & $2.2 \mathrm{yr}$ & $0-4.8$ & 6 & 45 & $77.5 \%$ & $58.9-96.1$ \\
& & & & & & & & \\
\hline
\end{tabular}

responses. This hypothesis is indirectly supported by the finding that many cancer therapeutics act by activating autophagy.

Signaling-lymphocytic-activation-molecule-family1 (SLAMF1) is the prototype member of a family of 9 genes that code for adhesion/costimulatory molecules that initiate signal transduction networks in T, natural killer, and antigen-presenting cells $(17,18)$. During T-B lymphocyte cross-talk, SLAMF1 works as a self-ligand and mediates a signal transduction pathway that enhances lymphocyte activation (19). In murine macrophages, SLAMF1 was found to control the killing of Gram-negative bacteria, by regulating phagosome functions through the recruitment of VPS34 and beclin1 (20).

In this work, we show that circulating $\mathrm{CD} 19^{+} / \mathrm{CD}^{+}$normal B-lymphocytes, considered the normal counterpart of CLL (21), constitutively express SLAMF1. SLAMF1 expression is maintained in CLL cells associated to a favorable prognosis. It is instead lost in a subset of patients who have an aggressive form of the disease characterized by shorter time to first treatment and OS. We also demonstrate that SLAMF1 activates the autophagic flux by modulating a signaling circuit that involves ROS generation, activation of JNK1/2, BCL2 phosphorylation, and ultimately association with beclin1 and VPS34 to stabilize the autophagic macrocomplex. In line with these observations, SLAMF1-deficient CLL cells are resistant to the action of autophagy-inducing therapies.

\section{Results}

SLAMF1 is heterogeneously expressed by CLL cells. Studies of surface antigen and gene expression profiling performed on limited sets of CLL patients showed that SLAMF1 is preferentially expressed by CLL cells bearing molecular markers of favorable prognosis (22-24).

In this work, SLAMF1 expression was evaluated in the CD19+ subset of a cohort of 300 CLL patients with a confirmed diagnosis of CLL and well-characterized clinical and molecular features (Supplemental Table 1; supplemental material available online with this article; doi:10.1172/JCI83013DS1). Expression was markedly heterogeneous with a median of $40 \%$ SLAMF1+ cells (Figure $1, \mathrm{~A}$ and C). This heterogeneity in expression was confirmed at the mRNA level (Supplemental Figure 1A). On the contrary, circulating CD19 ${ }^{+} / \mathrm{CD}^{+}$ B-lymphocytes from nonleukemic adult donors, considered the normal counterpart of CLL cells (21), were invariably SLAMF1 ${ }^{+}$(Figure $1, B$ and C, and Supplemental Figure 1A), with a median expression of $91 \%$, independently of CD27 expression (Supplemental Figure 1D) and in line with previous results (25). Forty-two patients were then tested for SLAMF1 expression on 2 different occasions at least 3 months apart (range 3-15 months), with no intervening therapy. Under these conditions, expression of the molecule was generally stable over time (Supplemental Figure 1B). Surface SLAMF1 showed a linear correlation with mRNA levels, in line with a direct relation- ship between gene transcription and surface expression of the protein $(r=0.7$, Supplemental Figure 1C).

These results indicate that, unlike normal circulating $\mathrm{CD} 9^{+} / \mathrm{CD}^{+} \mathrm{B}-\mathrm{lym}-$ phocytes, CLL cells express heterogeneous levels of SLAMF1, from which it is reasonable to infer that a proportion of CLL clones lost SLAMF1 expression during tumor transformation.

SLAMF1 expression is lost in a subset of patients with an aggressive form of CLL. The distribution of SLAMF1 was then analyzed according to clinical and molecular parameters. When the data was stratified based on the stage of the disease, it was apparent that SLAMF1 expression levels were markedly higher in stage A than in stage B and C patients combined (Figure 1D). Similarly, untreated patients were characterized by considerably higher SLAMF1 levels than treated ones, in line with the hypothesis that SLAMF1 marks a subset of patients with an indolent form of the disease. This difference was even more marked when considering patients who had not been administered therapy for at least 60 months, as opposed to those who received therapy within one year from diagnosis (Figure $1 \mathrm{E}$ ). These findings were confirmed by stratifying the cohort according to molecular markers. In each case, SLAMF1 levels were lower in the subset of patients bearing markers of unfavorable prognosis (such as the absence of somatic mutations in the IGHV genes or the expression of CD38 or CD49d) than in the counterpart (Figure 1F). Consistently, SLAMF1 levels were higher in patients with favorable cytogenetics (del13q14 as sole genetic abnormality or no abnormalities), as opposed to patients with del11q or del17p considered together (Figure $1 \mathrm{~F}$ ). Together, these data indicate that SLAMF1 expression clearly associates to a subset of patients with a more favorable prognosis.

By applying recursive-partitioning analysis, the optimal SLAMF1 cut-off within the CLL population under study was defined at 6\% (Supplemental Figure 2), with 233 patients considered

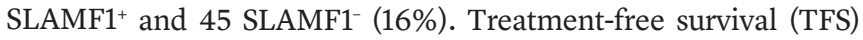
was significantly shorter for patients expressing SLAMF1 $\leq 6 \%$ cut-off, with a median TFS of 2.2 years versus 7.6 in patients who expressed SLAMF1 in > 6\% (Figure $1 \mathrm{G}$ and Table 1 ). Similar results were observed when considering overall survival (OS). Median OS was not reached, with a $77.5 \%$ survival rate at 10 years in SLAMF1 ${ }^{-}$ patients, compared with $94.7 \%$ in SLAMF1+ ones (Figure $1 \mathrm{G}$ and Table 1). Together, these results suggest that SLAMF1 expression is lost in a subset of CLL patients characterized by an aggressive disease with a shorter TFS and a lower OS.

SLAMF1 silencing modulates expression of genes involved in migration and intracellular vesicle formation. We then focused on the role of SLAMF1 in CLL cells by exploiting the cell line Mec-1, which was originally derived from a CLL patient and which is constitutively $\mathrm{SLAMF1}^{+}$. SLAMF1 was silenced using RNA interference (Mec-1/ SLAMF1 ${ }^{\text {sh }}$. Mec-1 cells were transfected with 3 SLAMF1-specific shRNAs (Supplemental Figure 3, A and B) and with a scrambled shRNA (Mec-1/Ctrl ${ }^{\text {sh }}$ ) and were selected by cell sorting and cloning by limiting dilution. Stably silenced clones were established from shSLAMF1\#1 and from a mixture of the 3 shRNAs. No differences in the expression of line-specific markers (CD19, CD20, CD23, 
A

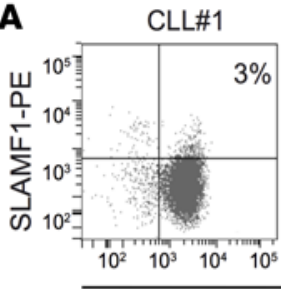

C

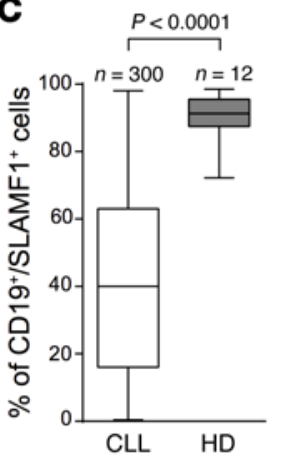

$\mathbf{F}$

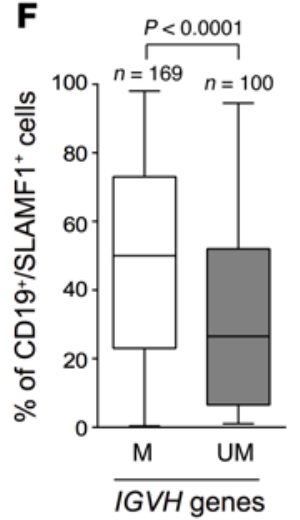

CLL\#2

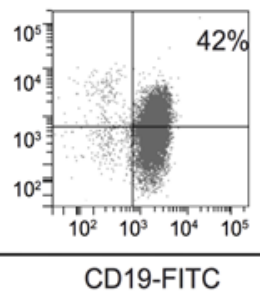

D

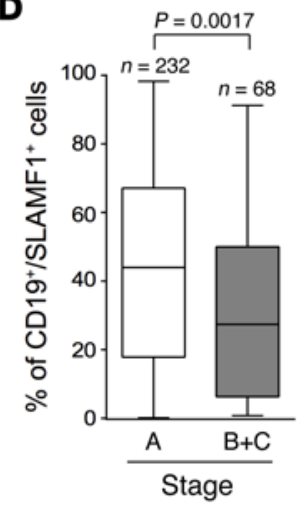

CLL\#3

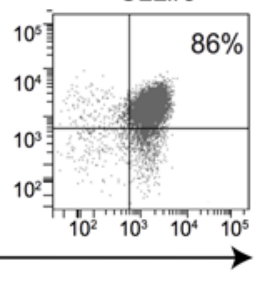

B

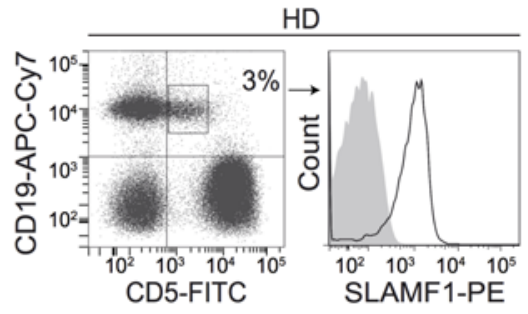

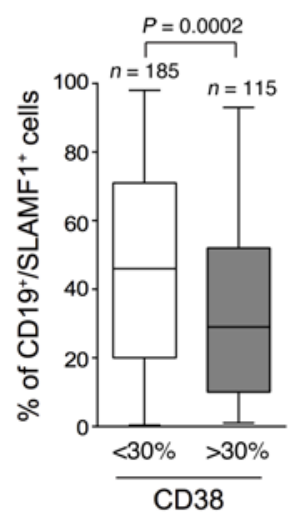

E
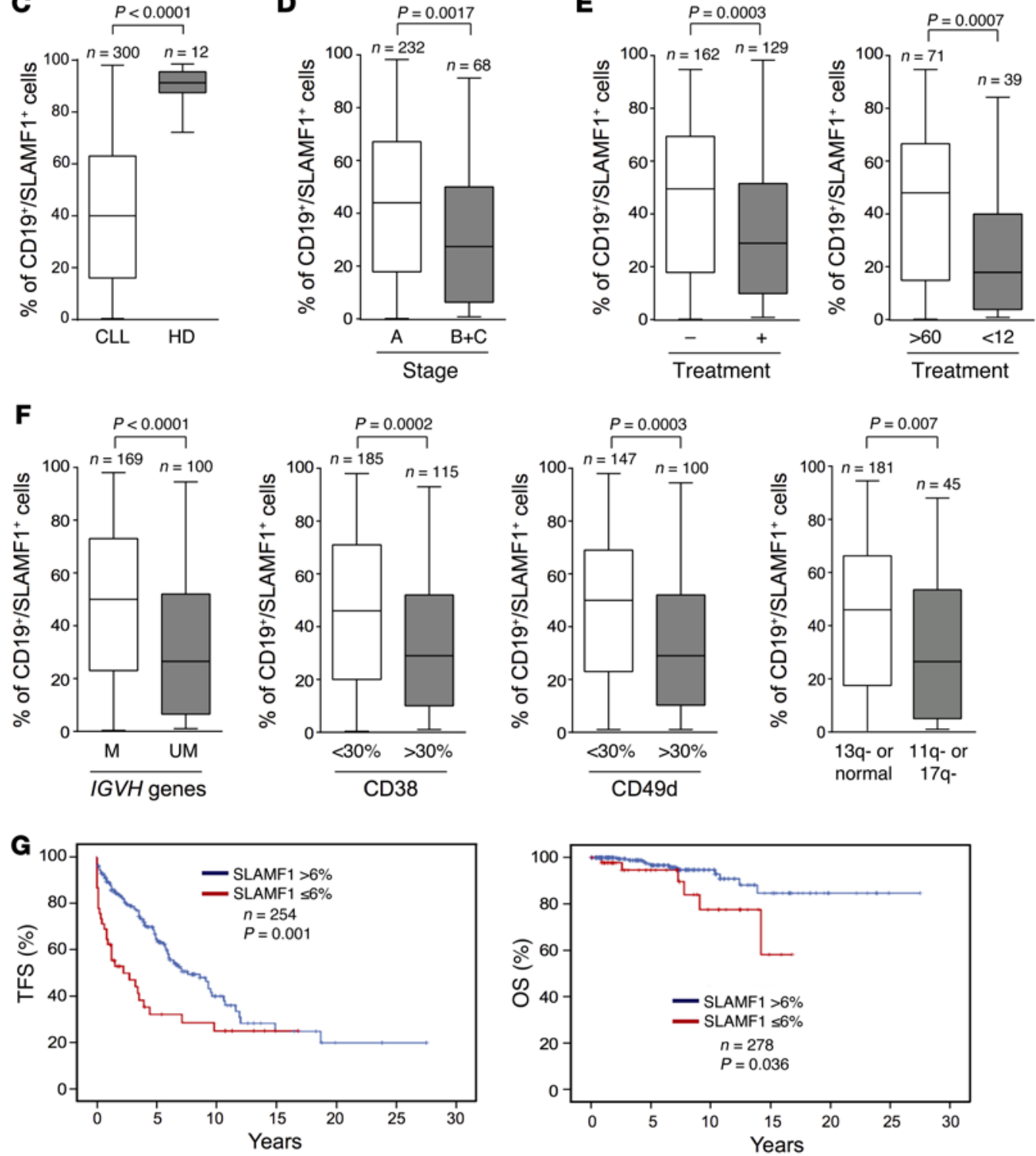

Figure 1. SLAMF1 expression is lost in a subset of CLL patients with a more aggressive form of the disease. (A) Dot plots showing CD19/SLAMF1 expression in 3 representative CLL patients. (B) SLAMF1 expression was analyzed in the CD19+/CD5+ fraction of PBMC preparations from normal donors. (C) Box plot showing cumulative data from 300 CLL patients and 12 normal donors. (D) Distribution of SLAMF1 expression according to disease stage or treatment conditions. Patients were grouped into stage $A$ versus stages $B+C$, according to the Binet classification. (E) Distribution of SLAMF1 expression according to treatment (chemotherapy or chemoimmunotherapy). --, untreated; +, treated with chemotherapy or chemoimmunotherapy. The box plot on the right considers patients who received no treatment for a period of at least 60 months, as opposed to patients who were treated within 1 year of diagnosis. (F) Distribution of SLAMF1 expression according to molecular and cytogenetic markers. The mutational status of the IGHV genes (unmutated if $>98 \%$ similar to the germline sequence) together with CD38 and CD49d expression were considered as molecular markers. The cut-off for CD38 and CD49d expression was $30 \%$. When considering cytogenetic abnormalities, patients were grouped on the basis of favorable (13q- or no abnormalities) vs. unfavorable (11qand 17p-) chromosomal deletions. (G) Kaplan Meyer curves showing the probability of TFS and OS of CLL patients categorized on the basis of SLAMF1 expression. The $6 \%$ cut-off was determined by recursive partitioning analysis. Statistical analyses (C-F) were performed using Mann-Whitney $U$ test. 
A
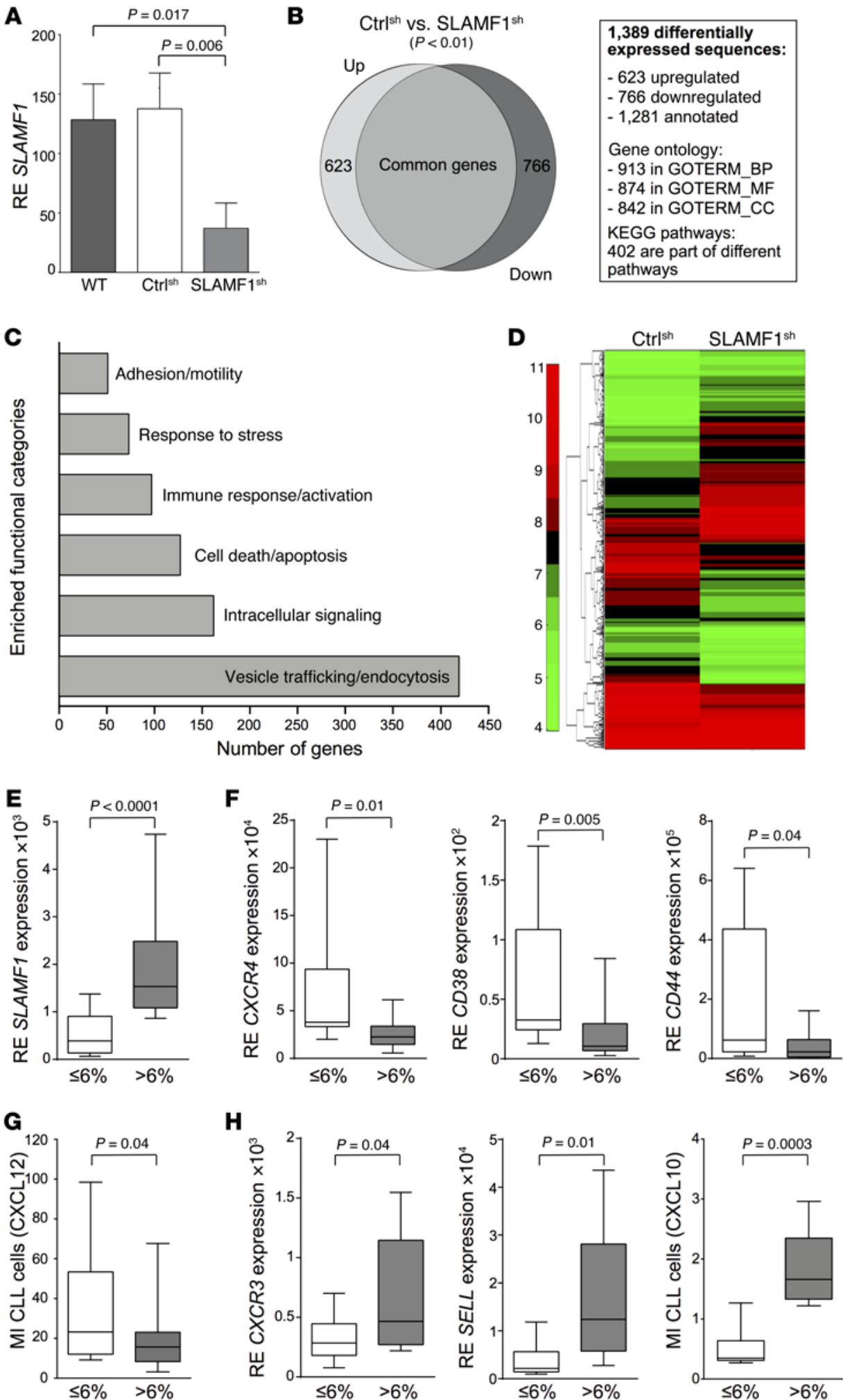

\section{H}
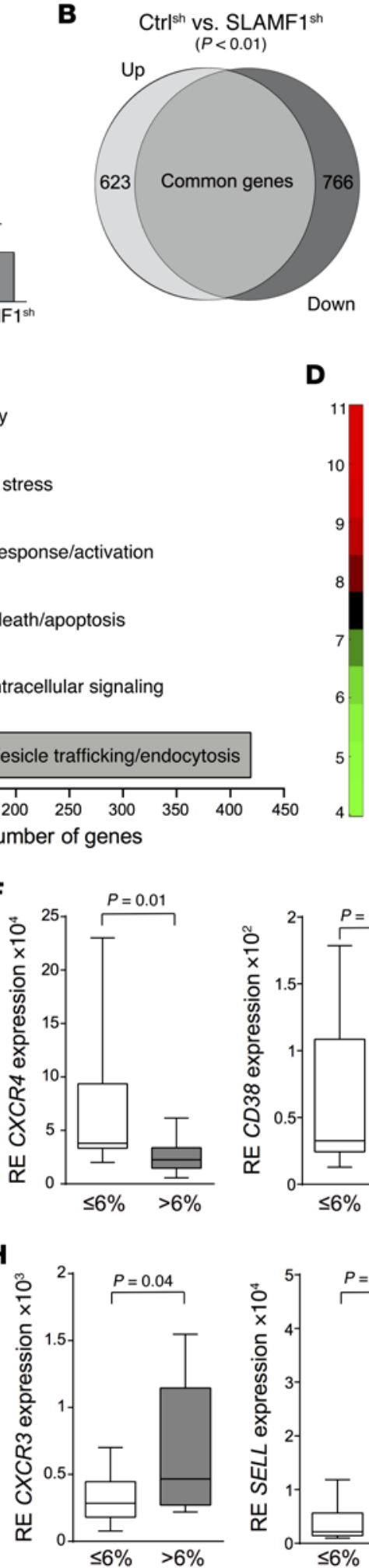

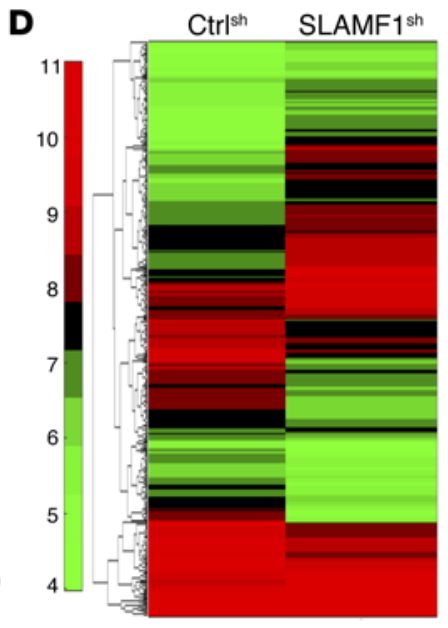

\begin{tabular}{|l|}
\hline 1,389 differentially \\
expressed sequences: \\
-623 upregulated \\
-766 downregulated \\
$-1,281$ annotated \\
\\
Gene ontology: \\
-913 in GOTERM_BP \\
-874 in GOTERM_MF \\
- 842 in GOTERM_CC \\
KEGG pathways: \\
402 are part of different \\
pathways
\end{tabular}

1,389 differentially
expressed sequences:

- 623 upregulated

766 downregulated

Gene ontology:

pathways

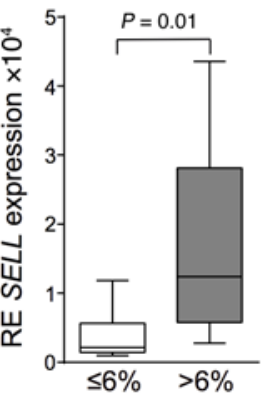

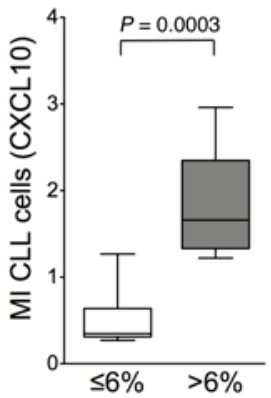

Figure 2. Loss of SLAMF1 expression is associated with the modulation of genetic pathways regulating cell movement and vesicle trafficking. (A) qPCR data showing stable downregulation of SLAMF1 mRNA in Mec-1 cells transfected with SLAMF1 shRNA and selected by sorting and limiting dilution cloning. Data from at least 5 independent experiments. (B) Comparison of the gene signature of Mec- $1 / \mathrm{Ctr}^{\text {sh }}$ vs. Mec- $1 / \mathrm{SLAMF}^{\text {sh }}$ cell clones. The light gray area in the Venn diagram identifies upregulated genes and the dark gray identifies downregulated genes in the Mec- $1 / \mathrm{Ctr}^{\text {sh }}$ vs. Mec- 1 / SLAMF1 ${ }^{\text {sh }}$. (C) Histogram plot showing the absolute number of genes modulated in the most representative functional categories identified by pathway-enrichment analysis. (D) Heat map of 419 transcripts differentially expressed in the comparison between Mec-1/Ctrl ${ }^{\text {sh }}$ and Mec-1/SLAMF1 ${ }^{\text {sh }}$ belonging to vesicle trafficking/endocytosis category. Red, increased expression; green, decreased expression. (E and $\mathbf{F}$ ) qPCR data showing expression of SLAMF1, CXCR4, CD38, and CD44 in purified CLL cells from SLAMF1 $(n=22)$ and SLAMF1- $(n=16)$ patients. (G) Results from chemotaxis experiments against CXCL12 of primary CLL cells derived from SLAMF1 $1^{+}(n=22)$ and SLAMF1- $(n=16)$ patients. (H) qPCR data showing expression of CXCR3 and SELL in purified CLL cells from SLAMF1 $1^{+}(n=22)$ and SLAMF1- $(n=16)$ patients. The graph on the right shows the results of a chemotaxis experiment against CXCL10 performed using primary CLL cells derived from SLAMF1+ $(n=6)$ or SLAMF1$(n=6)$ cells. MI, migration index, calculated as the ratio between the number of $\mathrm{CDS}^{+} /$ $\mathrm{CD}^{2}{ }^{+}$cells transmigrating in the presence and absence of CXCL12. RE, relative expression. Statistical analyses (A, E-H) were performed using Mann-Whitney $U$ test.
HLA-II) between the 2 cell lines or with the parental line were observed (not shown). Downregulation of SLAMF1 was confirmed using quantitative PCR (qPCR) (Figure 2A), immunofluorescence and Western blot (Supplemental Figure 3, C and D).

By applying global gene expression analysis, we observed that Mec-1/Ctrl ${ }^{\text {sh }}$ and Mec-1/SLAMF1 ${ }^{\text {sh }}$ have distinct profiles, with 623 upregulated and 766 downregulated sequences (Figure 2B). Inter- rogation of the 1,281 differentially expressed annotated genes using functional online tools showed marked enrichment for biological processes involved in vesicle trafficking/endocytosis, intracellular signaling, cell death/apoptosis, immune response/activation, response to stress and adhesion/motility (Figure 2, C and D).

Among these processes, we selected for further analyses the panel of genes in the adhesion motility category and those in the 
vesicle trafficking/endocytosis category. This choice was based on the existence of previous data functionally linking SLAMF1 to these processes. Mec-1/SLAMF1 ${ }^{\text {sh }}$ cells from each clone showed a significant difference in genes involved in cell migration, as compared with both $\mathrm{Mec}-1 / \mathrm{Ctrl}^{\mathrm{sh}}$ and Mec-1/WT. Upmodulation of CXCR4, the CXCL12 receptor, was confirmed both at the mRNA and protein levels (Supplemental Figure 4A). The CD38 ectoenzyme, which was found to associate with CXCR4 and to positively regulate CXCL12 signaling $(26,27)$, was similarly upregulated (Supplemental Figure 4B). Moreover, we found increased expression of CD44, an adhesion molecule that forms part of the CLL invadosome and that controls migration of CLL cells (Supplemental Figure $4 \mathrm{C}$ and refs. 28, 29). These data were confirmed by comparing primary CLL cells expressing SLAMF1 $\leq 6 \%$ or SLAMF1 $>6 \%$ cut-off, as also confirmed at the mRNA level (Figure 2E). In this cohort, CXCR4, CD38, and CD44 were significantly more expressed in the SLAMF1 $\leq 6 \%$ subset (Figure $2 \mathrm{~F}$ ). Consistent with this phenotype, Mec-1/SLAMF1 ${ }^{\text {sh }}$ cells showed higher chemotaxis toward CXCL12 than the Mec-1/Ctrl ${ }^{\text {sh }}$ cells (Supplemental Figure 4D). Likewise, when studying CXCL12-driven chemotaxis in a cohort of 38 primary CLL cases, those expressing SLAMF1 $\leq 6 \%$ showed a significantly higher migration index than the rest of the cohort (Figure 2G). Lastly, when analyzing chemotactic responses in primary CLL cells with a bimodal SLAMF1 expression, it was clear that the SLAMF $1^{\text {lo }}$ component showed more efficient chemotaxis toward CXCL12 than the SLAMF1 ${ }^{\text {hi }}$ one (Supplemental Figure 4E). Silencing of SLAMF1 in Mec-1 was also followed by a significant decrease, both at mRNA and protein levels, in CXCR3 and in CD62L (SELL) (Supplemental Figure 4, F and G), two molecules highly expressed in subsets of patients with a favorable prognosis $(30,31)$. Decreased CXCR3 and CD62L expression was confirmed in primary CLL cells (Figure $2 \mathrm{H}$ ). In line with this phenotype, Mec-1/ SLAMF1 $^{\text {sh }}$ cells and SLAMF1- primary CLL cells showed reduced migration toward CXCL10, as opposed to Mec-1/Ctrl ${ }^{\text {sh }}$ (Supplemental Figure $2 \mathrm{H}$ ) or SLAMF1+ primary CLL cells (Figure $2 \mathrm{H}$ ).

Of the 1,281 modulated genes, approximately one-third $(n=419)$ were part of the functional vesicle trafficking/endocytosis category. Among them, Mec-1/SLAMF1 ${ }^{\text {sh }}$ cells showed a marked downmodulation of vimentin (VIM), a cytoskeletal protein that can regulate protein trafficking and autophagy (32), and of VPS34 (PIK3C3), a member of the PI3K family responsible for autophagosome formation and accumulation (33) (Supplemental Figure 5, A and B). These differences were maintained in primary CLL cells and confirmed at the protein level (Figure 3A). Reconstitution of SLAMF1 expression by transfection of a cDNA encoding an shRNA-resistant human SLAMF1 (Supplemental Figure 5, C and D) was followed by rescued expression of vimentin and VPS34, further ruling out the possibility of off-target effects (Figure 3B).

Considered together, these data suggest that SLAMF1 silencing is followed by a significant upmodulation of proteins regulating migration and a downmodulation of molecules involved in intracellular vesicle formation, suggesting that these 2 biological processes are relevant in determining the inferior clinical performance of SLAMF1' patients.

SLAMF1 promotes the autophagic flux. The modulation of genes involved in vesicle formation, together with previous reports indicating that SLAMF1 regulates the assembly of the autophagic complex in mouse macrophages (20), prompted us to ask whether SLAMF1 activation could directly modulate autophagy in primary CLL cells. When autophagy is activated, the LC3B-I molecule (also known as Atg8) is cleaved, lipidated, and inserted as LC3B-II into the nascent autophagosome membrane. Cross-linking of SLAMF1 using an agonistic monoclonal antibody in purified CLL cells significantly increased endogenous LC3B aggregation and the amount of lipidated LC3B-II species when compared with untreated cells, as shown by confocal and by Western blot analyses (Figure 3, C and D). However, given that the autophagosome is an intermediate structure in a dynamic pathway, the number of autophagosomes observed at any given time is a function of the balance between the rate of their generation and the rate of their conversion into autolysosomes (34). Therefore, to determine the autophagic flux, chloroquine was used to block lysosomal acidification, leading to the inhibition of autophagosome turnover. The autophagic flux was represented as the difference between the intensity of the LC3B-II band in the SLAMF1 plus chloroquine condition versus the SLAMF1-alone condition or the chloroquine-alone versus the untreated condition (indicated in Figure $3 \mathrm{D}$ as $\triangle \mathrm{LC} 3-\mathrm{II}$ ), as reported (35). The finding of significantly increased amounts of LC3B-II species in SLAMF1-activated and chloroquine-treated primary CLL cells clearly showed that LC3B-II protein starts to accumulate upon SLAMF1 ligation (Figure 3D). The autophagosome then fuses with the lysosome, which can be visualized using the specific marker LAMP-2. The autophagolysosome can therefore be recognized by the simultaneous expression of LC3B and LAMP-2. LC3B puncta, which were clearly visible using confocal microscopy, almost completely overlapped with the lysosomal marker LAMP-2, confirming that the process is complete (Figure 3C). Thus, SLAMF1 promotes the autophagic flux in primary CLL cells. Similar results were observed using the Mec-1 cell line (Supplemental Figure 6, A and B). In addition, when using Mec-1 cells, the degradation of p62, a common target of the autophagic process, was apparent (Supplemental Figure 6B).

Electron microscopy analysis of primary CLL cells activated through SLAMF1 revealed the widespread appearance of electron-dense membrane-delimited bodies within the cytoplasm, with characteristic autophagic profiles. Some of them appeared to be early double-membrane autophagosomes (full arrow), while others - often close to multivescicular bodies (arrowhead) - seemed to be late autophagosomes (open arrow). The latter were more visible after SLAMF1 cross-linking and were delimited by a single membrane, with electron-dense content, including partially degraded ribosomes, showing up as dark granular clumps (Figure 3E).

These results demonstrate that ligation of SLAMF1 activates the autophagic process both in primary cells and in a reference cell line.

Molecular mechanisms behind SLAMF1-driven autophagy. Autophagy can be initiated by a number of signals and conditions, but the cellular redox status is believed to play a critical role (36). Cross-linking of SLAMF1 with an agonistic mAb induced ROS accumulation, commencing 30 minutes after antibody binding and reaching its maximal accumulation after 18 hours in primary CLL cells (Figure 4A). Specifically, we used flow cytometry to measure the amount of intracellular hydrogen peroxide $\left(\mathrm{H}_{2} \mathrm{O}_{2}\right)$ with the 5-(and-6)-chloromethyl-2,7-dichlorodihydrofluorescein diacetate (CM- $\mathrm{H}_{2}$ DCFDA). CM-H DCFDA is a widely used indicator of oxi- 

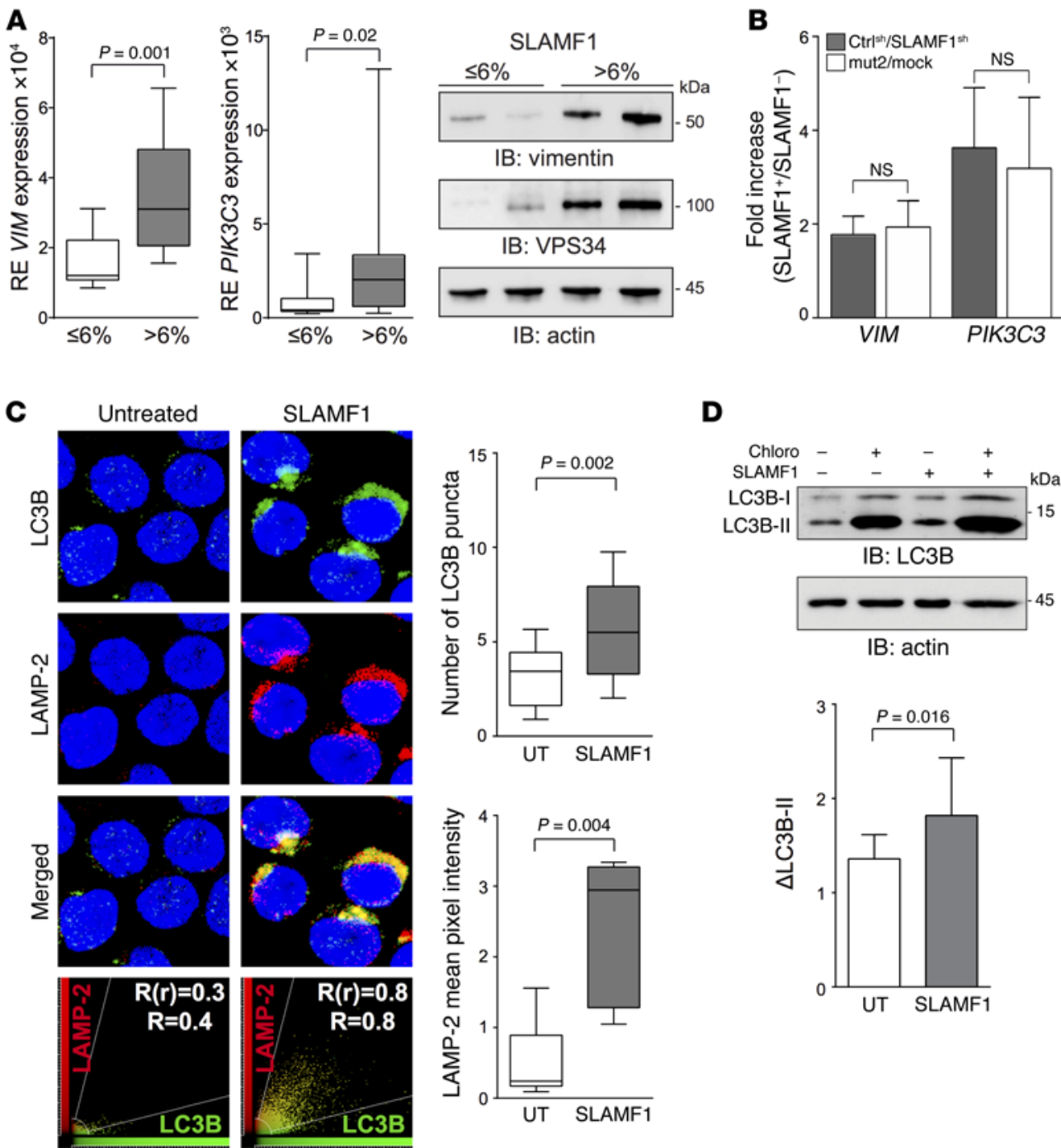

D

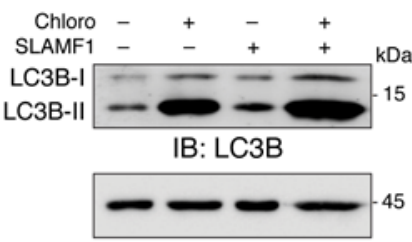

IB: actin
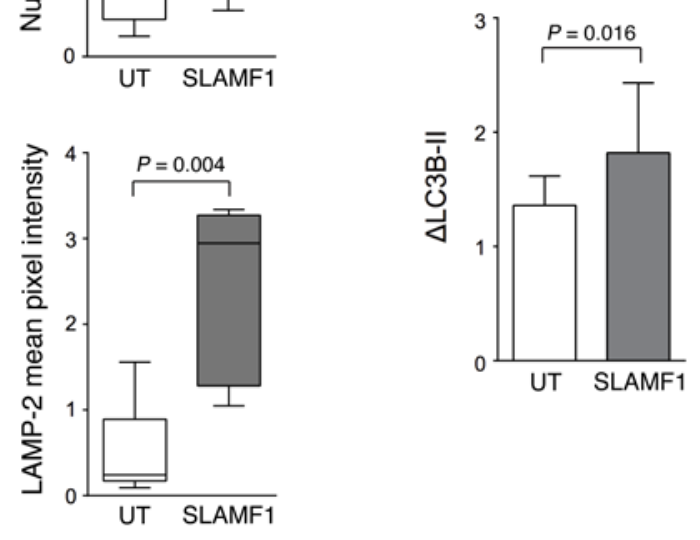

Figure 3. Cross-linking of SLAMF1 in CLL cells stabilizes the autophagic flux. (A) qPCR and Western blot showing vimentin and VPS34 mRNA and protein expression by Western blot in primary SLAMF1+ $(n=9)$ or SLAMF1- $(n=14)$ CLL cells. (B) Reconstitution of SLAMF1 expression in Mec-1/SLAMF1 ${ }^{\text {sh }}$ cells by transfection of a shRNA-resistant SLAMF1 mutant (mut2) or an empty vector (mock). Data are presented as the ratio between expression of the target gene in Mec- $1 / \mathrm{Ctr}^{\text {sh }}$ cells over that of Mec-1/SLAMF1 ${ }^{\text {sh }}$ cells (gray bars) or the ratio between Mec-1/ SLAMF1 ${ }^{\text {sh }} /$ mut2 cells over Mec- $1 /$ SLAMF $1^{\text {sh }} /$ mock transfected cells (open bars). The graph shows results from 4 independent transfection experiments. (C) Confocal microscopy analysis of LC3B (green) and LAMP-2 (red) staining in purified CLL cells treated with the agonistic antiSLAMF1 monoclonal antibody A12, followed by a cross-linker (6 hours, $37^{\circ} \mathrm{C}$ ). Nuclei were counterstained with DAPI (blue). Original magnification, $\times 63$; zoom factor of 2 . Representative images from 5 different experiments. Scatter plots represent colocalization analyses between LC3B and LAMP-2 using LAS AF Version Lite 2.4 software. Pearson coefficient $(R)$ and Overlap coefficient $(R[r])$ are listed. The box plot shows cumulative numbers of LC3B puncta (calculated as average number of puncta/cell) in purified CLL cells from 5 different patients. LAMP-2 mean pixel intensity was analyzed using Image] software. (D) Western blot analysis of LC3B-I/II expression, following SLAMF1 cross-linking in the presence or absence of chloroquine (Chloro, 15 $\mu \mathrm{M}) . \Delta \mathrm{LC} 3 \mathrm{~B}-\mathrm{Il}$ levels were calculated as the difference of LC3B-II protein levels between chloroquine-treated and untreated states. Data from 8 independent experiments. (E) Electron microscopy analysis of CLL cells treated with SLAMF1 antibody as in C. Upon SLAMF1 ligation, autophagosomes became visible as double membranes surrounding cytoplasmic material (full arrow). Late autophagosomes were visualized as a single membrane surrounding a more electron-dense interior (open arrow), in proximity to multivesicular bodies (MVB, arrowhead). Scale bars: $0.5 \mu \mathrm{m}$. UT, untreated. Mann-Whitney $U$ test (A, C) or Wilcoxon signed rank test (D) were used. 
A
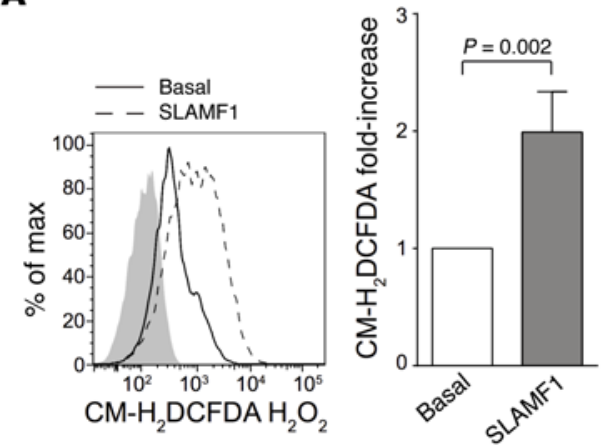

B

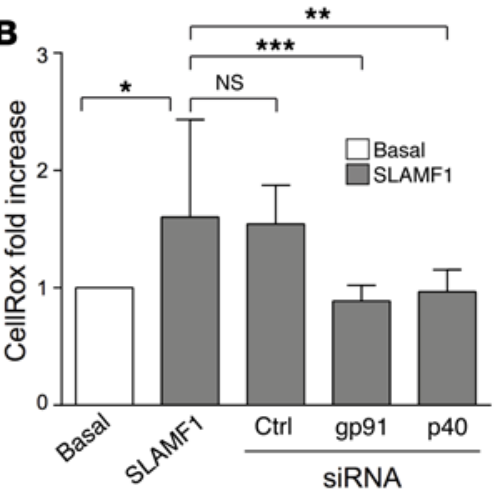

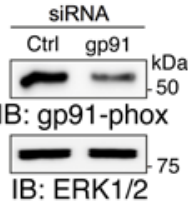

Ctrl p40

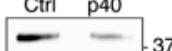

IB: p40-phox

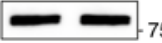

IB: ERK1/2

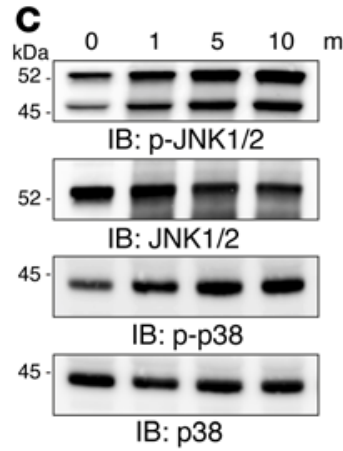

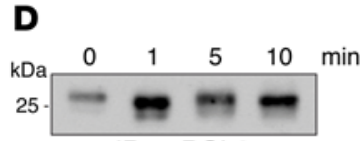

IB: p-BCL2

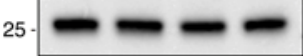

IB: BCL2
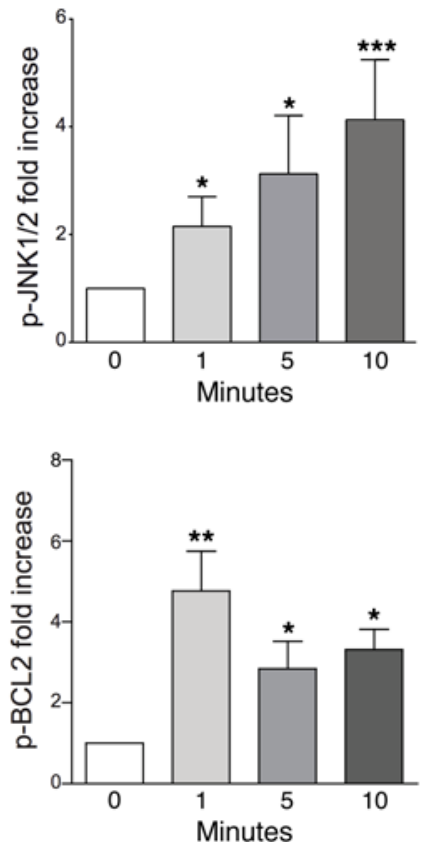

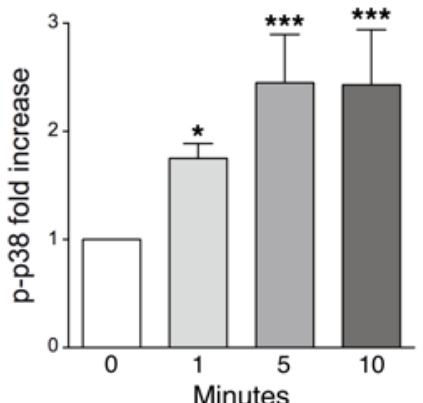

E

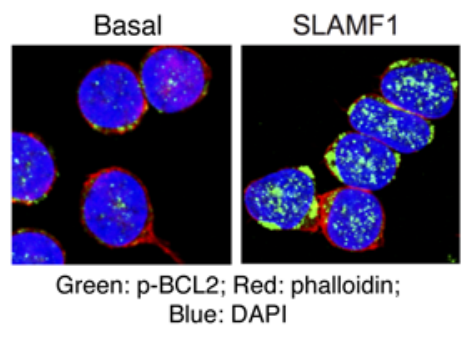

Figure 4. SLAMF1 cross-linking induces ROS accumulation, activates JNK1/2 and p38 kinases, and phosphorylates BCL2. (A and B) SLAMF1 cross-linking induces ROS accumulation, as measured by flow cytometry in CLL cells (A) or Mec-1 cells (B) by using CM-H DCFDA or CellRox dies, respectively. The histogram on the left shows results from a representative experiment. The bar graph in $\mathbf{A}$ derives from 8 independent experiments using primary cells from 8 different patients. Silencing of gp91-phox or p40-phox of the NOX2 enzyme complex by siRNA transfection impairs ROS accumulation following SLAMF1 cross-linking in Mec-1 cells (B). The Western blot confirms silencing of both molecules. Results from 5 independent experiments, each performed in triplicate. (C) Ligation of SLAMF1 on primary CLL cells induces time-dependent tyrosine phosphorylation of JNK1/2 and p38. A representative blot is shown, together with cumulative data obtained from 8 independent experiments from 8 different patients. The fold-increase represents the increase in band intensity over the untreated condition. (D) Ligation of SLAMF1 induces a rapid and robust phosphorylation of BCL2 at S70 in primary CLL cells, as shown with a specific mAb. The bar graph shows cumulative data from 7 different patients. (E) Confocal microscopy showing p-BCL2 accumulation (green) following SLAMF1 ligation in primary CLL cells. The cytoskeleton was visualized by AlexaFluor 568-conjugated phalloidin; nuclei were counterstained with DAPI. Original magnification, $\times 63$; zoom factor of 2 . Representative image from 5 independent experiments. ${ }^{* * *} P \leq 0.001,{ }^{*} P \leq 0.01$, and ${ }^{*} P \leq 0.05$, as calculated by Friedman's test followed by Dunn's multiple comparison.

dative stress (37) and becomes fluorescent upon oxidation by $\mathrm{H}_{2} \mathrm{O}_{2}$ or free radicals downstream. These results were confirmed in the Mec-1 cell line with the CM- $\mathrm{H}_{2}$ DCFDA probe (Supplemental Figure 7A) and with the CellROX assay (Figure 4B), also suitable for $\mathrm{GFP}^{+}$cells. ROS production following SLAMF1 cross-linking was impaired after silencing of gp91-phox or p40-phox subunits of the NADPH oxidase 2 (NOX2) complex (Figure 4B and Supplemental Figure 7B), confirming that NOX2 is responsible for SLAMF1induced ROS generation, as observed in other models $(20,38)$.

Activation of the MAP kinases, particularly of JNK1/2 and of $\mathrm{p} 38$, is critical during autophagic execution and has been directly linked to an increase in intracellular ROS production $(39,40)$. Cross-linking of SLAMF1 was followed by a prominent tyrosine phosphorylation of JNK1/2 (p46 and p54 isoforms, Thr183/Tyr185) and of p38 (Thr180/Tyr182), which started after 1 minute and peaked after 10 minutes, with highly reproducible kinetics in the 8 patients studied (Figure 4C). Overlapping results were obtained after SLAMF1 activation in Mec-1 cells (Supplemental Figure 7C).

The execution of autophagy relies on the assembly of the autophagosome macrocomplex, which contains the scaffold protein beclin1, the enzyme VPS34 and UVRAG, and various other proteins $(41,42)$. However, under steady state conditions, beclin1 


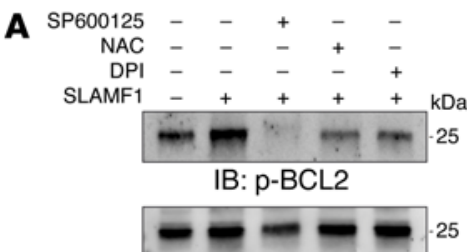

IB: BCL2

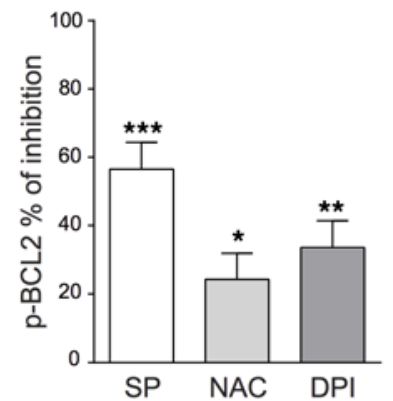

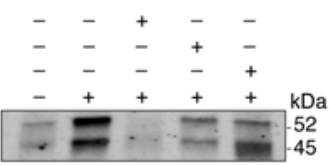

IB: $\mathrm{p}-J \mathrm{NK} 1 / 2$

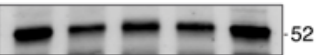

IB: JNK1/2

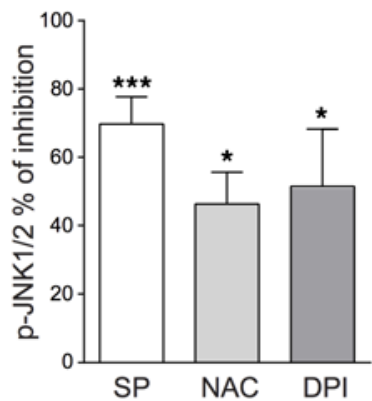

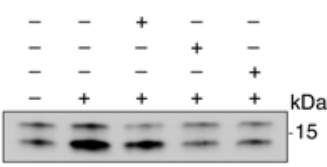

IB: LC3B

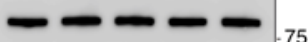

IB: ERK1/2

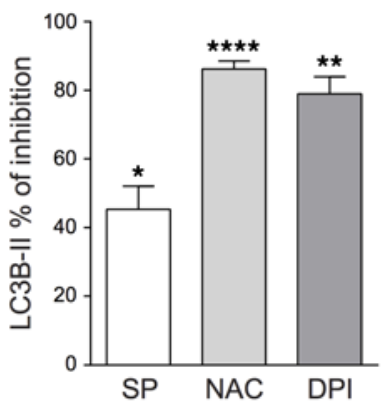

B

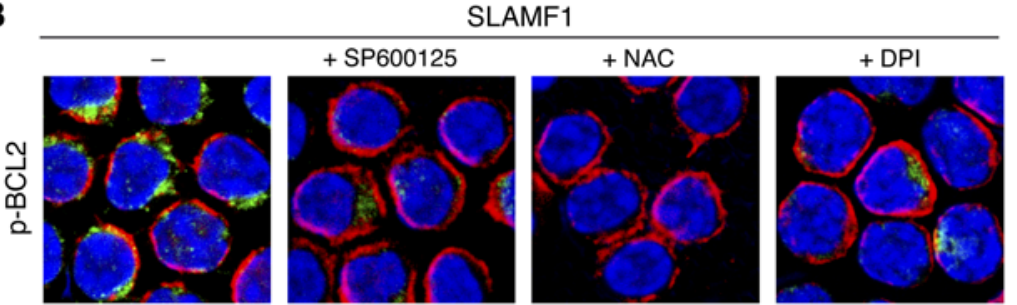

Green: p-BCL2; Red: phalloidin; Blue: DAPI

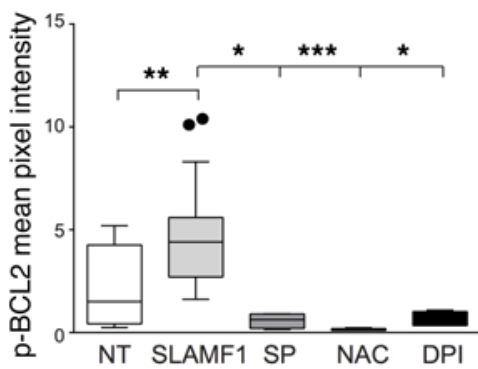

C

SLAMF1
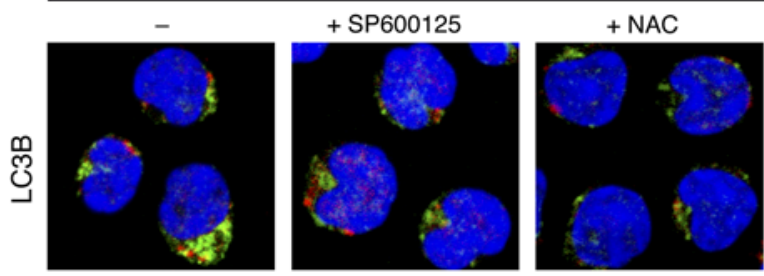

Green: LC3B; Red: LAMP-2; Blue: DAPI
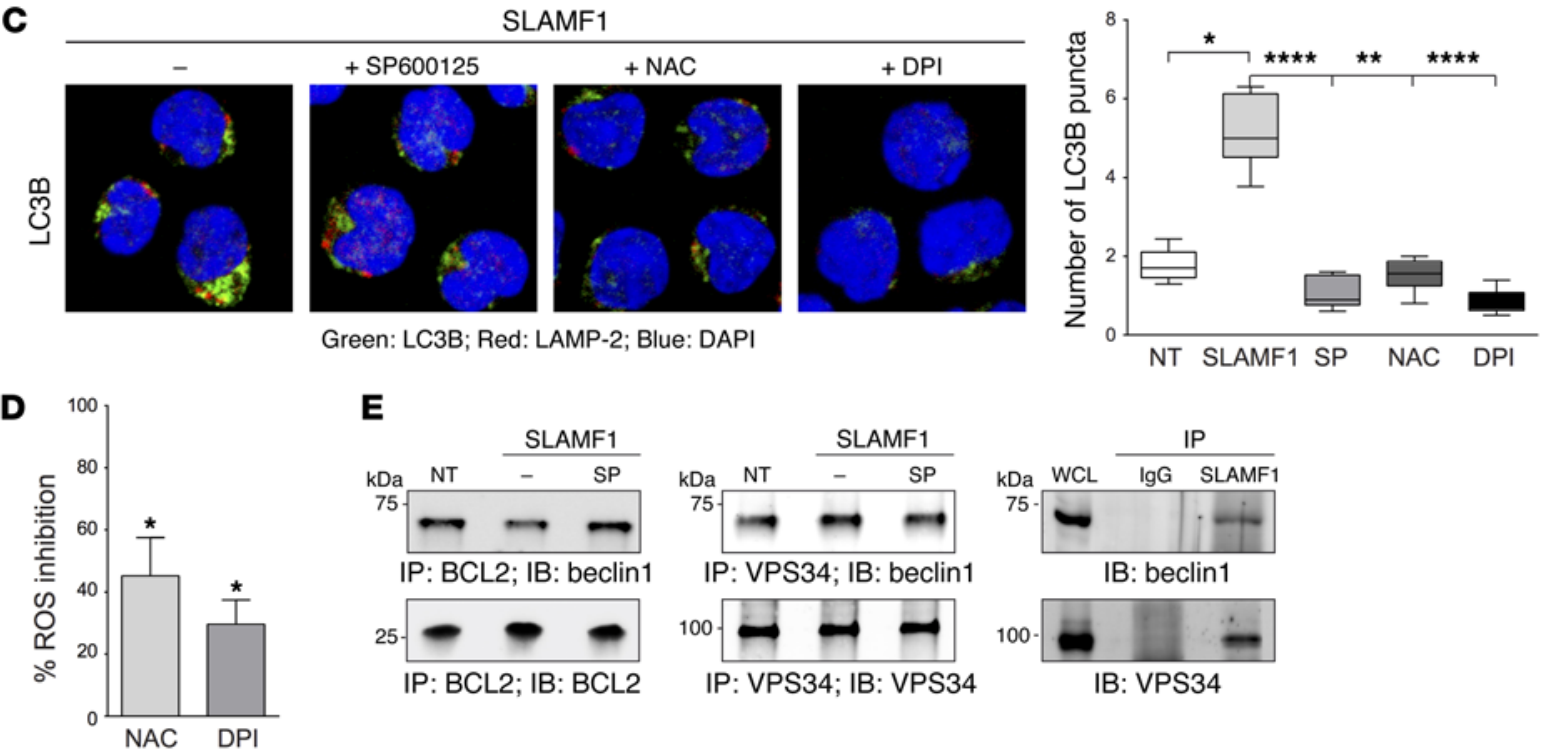

E
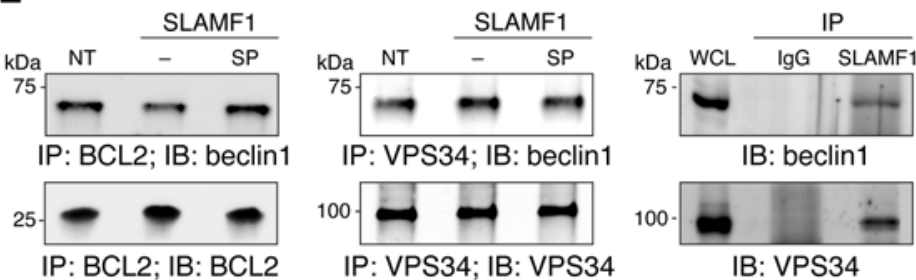

IB: VPS34

Figure 5. Use of selective inhibitors to highlight sequential steps in the SLAMF1-induced autophagy. (A) Effects of the pretreatment with SP600125 (JNK1/2 inhibitor, $15 \mu \mathrm{M}$ ), NAC (ROS scavenger, $5 \mathrm{mM}$ ), or DPI (NOX2 inhibitor, $0.05 \mu \mathrm{M}$ ) in the phosphorylation of BCL2, JNK1/2, and the appearance of lipidated LC3B induced upon SLAMF1 ligation in Mec-1 cells. Representative gel from 8 different experiments. The percentage of inhibition was calculated as: 100 (intensity of phosphorylated protein/control protein), after considering $100 \%$ of the value obtained in the SLAMF1-alone condition. (B and C) Confocal microscopy of primary CLL cells showing the effects of SP600125 $(5 \mu \mathrm{M})$, NAC ( $2.5 \mathrm{mM})$ and DPI $(0.05 \mu \mathrm{M})$ on the phosphorylation of BCL2 (B) and on LC3B (C) induced upon SLAMF1 ligation. Original magnification, $\times 63$; zoom factor of 2 . The box plot shows mean pixel intensity of BCL2 signal or cumulative numbers of LC3B puncta (calculated as average number of puncta/cell) in purified CLL cells from 3 different patients treated as described. (D) Bar graph showing the percentage of inhibition in ROS accumulation following SLAMF1 cross-linking in the presence of NAC and DPI, considering the level of ROS in the SLAMF1-alone condition to be $100 \%$. ROS accumulation was measured with the CellROX probe. (E) Immunoprecipitation experiments performed in Mec-1 cells after cross-linking SLAMF1 with the A12 antibody (30 minutes, $37^{\circ} \mathrm{C}$ ). Where indicated, SP600125 (SP) was added. The right image is representative of an immunoprecipitation experiment with an anti-SLAMF1 or an isotype-matched irrelevant antibody and subsequent immunoblot for beclin1 and VPS34. Whole cell lysate (WCL) is shown in the first lane. Representative images from 3 independent experiments. Statistical significance was calculated using Friedman's test followed by Dunn's multiple comparison. ${ }^{* * * *} P \leq 0.0001$, ${ }^{* * *} P \leq 0.001,{ }^{* *} P \leq 0.01$, and ${ }^{*} P \leq 0.05$. 
is bound to the antiapoptotic protein BCL2 and is consequently inactive $(33,43,44)$. Phosphorylation of critical threonine and serine residues in BCL2 triggers a conformational change in the molecule, which leads to its dissociation from beclin1 $(45,46)$. SLAMF1 cross-linking was followed by a prominent phosphorylation of BCL2 at serine 70 (S70) (Figure 4D), which was already evident after 1 minute of receptor engagement. This result was obtained using patient-derived cells and confirmed in the Mec-1 cell line model (Supplemental Figure 7D). BCL2 phosphorylation could also be confirmed using confocal microscopy, clearly indicating that the molecule is still phosphorylated 6 hours after SLAMF1 cross-linking, consistent with the appearance of autophagic vesicles (Figure 4E and Supplemental Figure 7E).

These findings suggest that a possible mechanism connecting SLAMF1 to the autophagy machinery relies on generation of ROS and activation of JNK1/2, which would then phosphorylate BCL2, dissociating it from beclin1 and leaving it free to assemble the autophagic complex. Proof of concept was obtained by using a JNK1/2 inhibitor, a ROS scavenger, and DPI as a specific NOX2 inhibitor. BCL2 phosphorylation was blocked after treatment with the JNK1/2 inhibitor SP600125, the ROS scavenger NAC, and the NOX2 inhibitor DPI (Figure 5A). Inhibition of BCL2 phosphorylation was maintained for at least 6 hours, as shown by confocal microscopy using primary CLL cells (Figure 5B). All 3 inhibitors interfered with JNK1/2 phosphorylation and with the activation of autophagy, as determined by Western blot and by confocal microscopy (Figure 5C and Supplemental Figure 8, A and $\mathrm{B}$ ), confirming that BCL2 phosphorylation is critical for the induction of SLAMF1-triggered autophagic flux. The finding of impaired ROS production upon DPI treatment confirmed that NOX 2 contributes, at least in part, to ROS production in SLAMF1treated CLL cells (Figure 5D).

Coimmunoprecipitation experiments showed that SLAMF1 cross-linking was followed by a marked dissociation of the BCL2/ beclin1 complex. Incubation with the JNK1/2 inhibitor SP600125 substantially prevented BCL2/beclin1 dissociation, suggesting that this is the main molecular mechanism involved in the activation of autophagy (Figure 5E). Simultaneously, upon SLAMF1 ligation, there is an increased association of beclin1 to VPS34, partially abrogated by inhibiting JNK1/2 (Figure 5E). Lastly, SLAMF1 was found to interact directly with the autophagic complex, as inferred from the association between SLAMF1, VPS34, and beclin1 (Figure 5E).

$S L A M F 1^{-}$cells are more resistant to autophagy-inducing therapies. Besides maintaining cellular homeostasis under normal growth conditions, autophagy critically controls cellular responses to stressful conditions $(47,48)$. We therefore investigated whether loss of SLAMF1 affects responses to drugs known to activate autophagy, such as fludarabine or the $\mathrm{BH} 3$ mimetic ABT-737. Fludarabine is a commonly used chemotherapeutic agent in CLL (49), while ABT-737 is the prototype of a novel family of molecules that activate autophagy by causing the dissociation of the BCL2/beclin1 complex (50).

Exposure of Mec-1/Ctrlsh or Mec-1 WT cells to fludarabine or ABT-737 was followed by a sharp increase in ROS levels and by the activation of autophagy (Figure 6, A and B). Immunostaining analysis for LC3B in Mec-1/Ctrl ${ }^{\text {sh }}$ cells treated with fludarabine or with ABT-737 for 5 hours showed an increase in the number of LC3B puncta and a complete execution of the autophagy flux, as demon- strated by the colocalization between LC3B and LAMP-2 and by the degradation of p62 (Figure 6, B and C). In contrast, Mec-1/ SLAMF1 ${ }^{\text {sh }}$ showed limited increase in ROS levels, with no modulation of the autophagic flux or p62 degradation (Figure 6, A-C). Consequently, treatment of $\mathrm{Mec}-1 / \mathrm{Crl}{ }^{\text {sh }}$ cells with both drugs used at therapeutic concentrations induced apoptosis, while Mec-1/ SLAMF1 ${ }^{\text {sh }}$ appeared resistant (Figure 6D). These results link SLAMF1 to the activation of drug-induced autophagy in CLL cells.

Confirmation was obtained using primary cells, where SLAMF1 ${ }^{\text {hi }}$ CLL cells underwent significantly more apoptosis than SLAMF1 ${ }^{\text {lo }}$ ones in response to ABT-737 (Figure 6E). Likewise, apoptosis in response to fludarabine was markedly higher in SLAMF1 $1^{\text {hi }}$ than in SLAMF1 ${ }^{\text {lo }}$ cells. These results were confirmed after sorting the same primary CLL clones with a bimodal SLAMF1 distribution, allowing for comparison of SLAMF ${ }^{\text {hi }}$ and SLAMF1 ${ }^{\text {lo }}$ cells with the same clonal origin (Figure 6E). Results from 5 different patients make it clear that the SLAMF $1^{\text {lo }}$ component of the clone is significantly more resistant to apoptosis than its counterpart (Figure 6E). No patients with del17p were included in this study, to eliminate confounding elements.

Together, our results demonstrate that loss of SLAMF1 expression from the cell surface affects spontaneous and druginduced autophagy responses, making these cells resistant to therapy and potentially explaining the unfavorable clinical behavior of SLAMF1-deficient CLL clones.

\section{Discussion}

In cancer, autophagy can participate in both tumor suppression and progression mechanisms. Depending on the cellular context, autophagy may enable tumor cells to survive chemotherapy-mediated stress, or it may maintain cellular homeostasis by removing damaged organelles and preventing the genomic damage that leads to cancer (51-53). In leukemia, several studies have shown that autophagy is activated upon treatment with different chemotherapeutic agents, inducing cell death. This is true also for CLL, where many currently used drugs - including fludarabine, dexamethasone, idelalisib, and the novel BCL2 antagonists (54-56) - have been suggested as having an autophagy-mediated effect.

This work connects the expression of the surface receptor SLAMF1 to the modulation of chemotactic responses, providing support to the hypothesis that the ability of cells to localize in a growth-favorable environment conditions disease aggressiveness. The second observation of this work is that SLAMF1 regulates an intracellular autophagic pathway, which is in turn important in dictating therapeutic responses. In agreement with previous observations (25), our findings confirm that normal B-lymphocytes uniformly express SLAMF1 at high levels, including the CD19+/ $\mathrm{CD}^{+}$subset. However, as expected on the basis of gene expression profiling studies (22-24), SLAMF1 expression in the patient cohort studied was highly variable. Reduced SLAMF1 levels typically associated with clinical or molecular markers of unfavorable prognosis, including a more advanced stage of disease, absence of mutations in the IgV genes, and surface expression of CD38 and of CD49d. As such, the patient subset expressing SLAMF1 $\leq 6 \%$ ( $\approx 15 \%$ of the cohort examined) was characterized by a significantly shorter time to first treatment and OS. It is important to note that SLAMF1' ${ }^{-}$patients needed therapy significantly earlier 
A

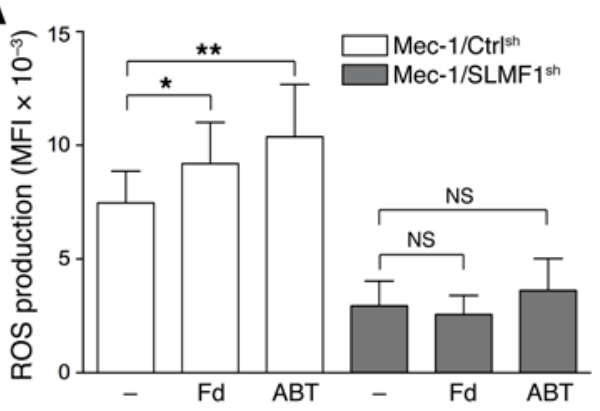

B

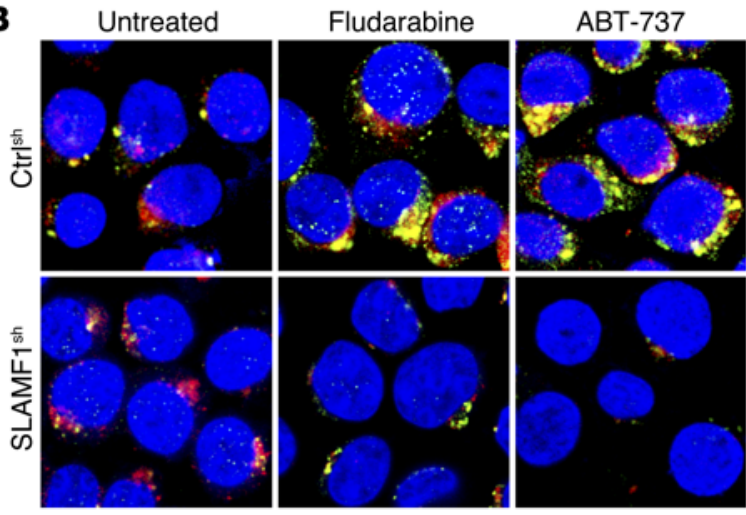

Green: LC3B; Red: LAMP-2; Blue: DAPI
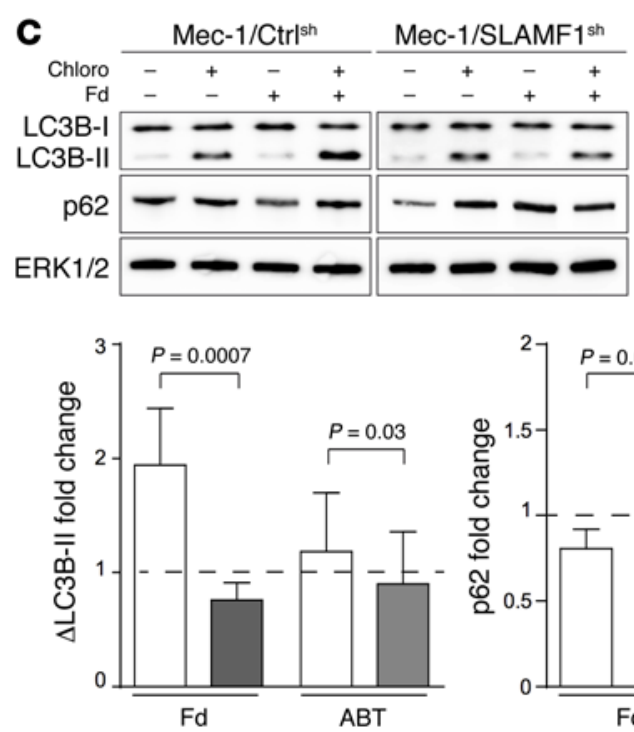

$\mathbf{E}$

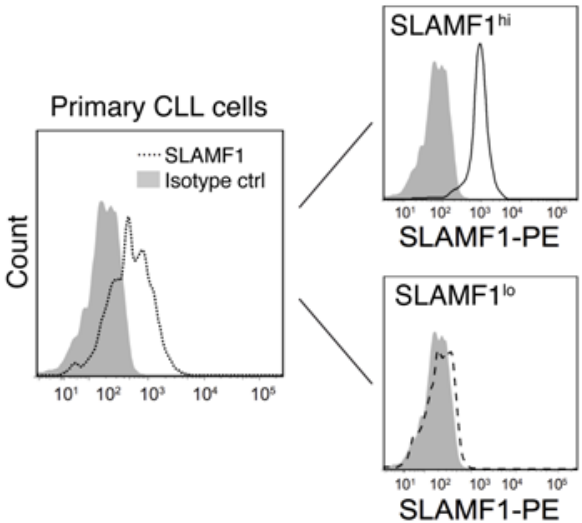

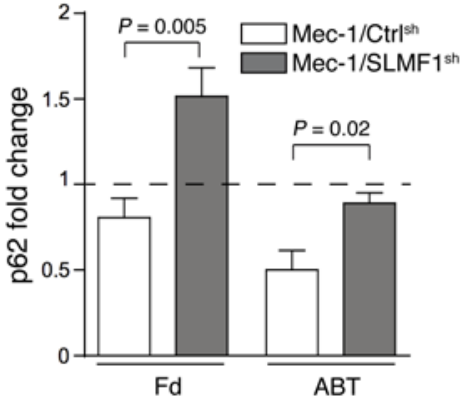
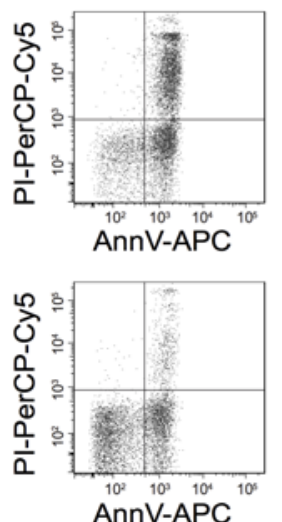

Mec-1/SLAMF1 $1^{\text {sh }}$
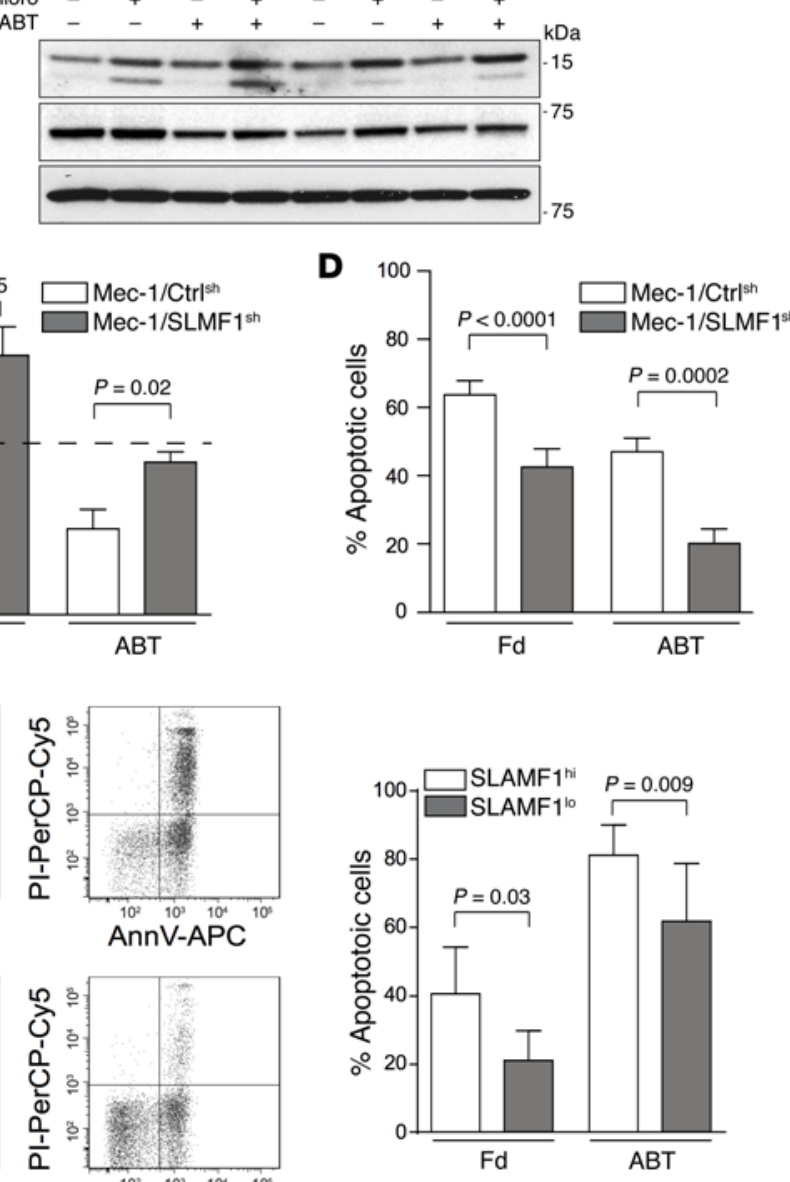

Figure 6. SLAMF1-deficient cells are resistant to drugs that activate autophagy. (A) ROS production upon 5 hour culture of Mec-1/Ctrlsh (white bars) and Mec-1/SLAMF1 ${ }^{\text {sh }}$ (gray bars) in the presence of fludarabine $(100 \mu \mathrm{M})$ or ABT-737 $(10 \mu \mathrm{M})$. ROS were measured using the CellROX assay and are shown as mean fluorescence intensity (MFI) values. Statistical significance was calculated using Friedman's test followed by Dunn's multiple comparison using data from 5 independent experiments performed in triplicate. ${ }^{*} P<0.05$; ${ }^{* *} P<0.01$. (B and $\mathbf{C}$ ) Induction of autophagy after 5 -hour cultures with fludarabine or ABT-737 was shown in Mec-1/Ctrlsh and Mec-1/SLAMF1 ${ }^{\text {sh }}$ by staining for LC3B (green) and LAMP-2 (red), followed by confocal microscopy analysis (original magnification, $\times 63$; zoom factor of 2) (B) or by Western blot for LC3B and p62 (C). Cells were pretreated with chloroquine for 1 hour to block the autophagic flux. ERK1/2 was used as internal loading control. $\triangle$ LC3B-II was calculated as indicated in Figure 3D. The fold-change was calculated over the untreated condition. The p62 fold-change was calculated after normalizing p62 over ERK1/2, with the untreated condition set to 1. Data from 6 experiments. (D) Bar graph showing apoptosis levels in Mec-1/Ctrlsh and Mec-1/SLAMF1 ${ }^{\text {sh }}$ cultured in the presence of fludarabine (100 $\left.\mu \mathrm{M}\right)$ or ABT-737 (10 $\left.\mu \mathrm{M}\right)$ for 48 hours. The percentage of apoptosis was calculated as the sum of cells stained by Annexin $V$ and by propidium iodide (PI). (E) Primary CLL cells $(n=5)$ with a bimodal distribution of SLAMF1 were separated by cell sorting in a SLAMF1 ${ }^{\text {hi }}$ and a SLAMF1 ${ }^{10}$ subset. The 2 sorted subpopulations were cultured for 24 hours in the presence of fludarabine $5 \mu \mathrm{M}$ or ABT-737 $(10 \mathrm{nM})$, and cell death was determined by Annexin V/PI staining. Statistical analyses were performed using Mann-Whitney test. 
than their counterparts, providing an initial link between SLAMF1 expression and treatment requirement. The conclusion from this first part of the work is that a subset of CLL patients with clinical and molecular hallmarks of aggressive disease is characterized by reduced SLAMF1 expression. The finding of a direct relationship between the percentage of SLAMF1+ leukemic cells and the number of mRNA copies favors the hypothesis of a transcriptional downregulation of the gene in the negative subset of patients, even if the molecular mechanism is currently unknown.

The analysis of the transcriptome of Mec- 1 cells with a stably silenced SLAMF1 showed that a significant number of the modulated genes are involved in cytoskeletal movements and in vesicle trafficking. These modifications were maintained at the protein level, suggesting functional implications; in fact, Mec-1/ SLAMF1 ${ }^{\text {sh }}$ cells migrated significantly more in response to CXCL12 and significantly less in response to CXCL10. Increased expression of the CXCR4 receptor and of CD38, a surface enzyme that enhances chemotactic responses (57), on the one side and decreased expression of CXCR3 on the other provide a partial explanation to this phenomenon. Considering the marked differences between the Mec-1 cell line and primary CLL cells in terms of phenotype (e.g., lack of CD5 expression in the cell line) and growth properties (e.g., exponentially growing line with a duplication time of about 20 hours), we confirmed these findings in primary cells. By analyzing the migration index of a cohort of CLL patients, it became clear that samples with expression of SLAMF1 $\leq 6 \%$ migrated significantly more toward CXCL12. This difference was maintained when comparing the SLAMF1 ${ }^{\text {hi }}$ versus SLAMF ${ }^{\text {lo }}$ components of the same CLL clone, which share a genetic structure. Likewise, primary SLAMF1 ${ }^{\text {lo }}$ primary CLL cells migrated less in response to CXCL10, a feature that has been linked to a favorable clinical behavior of the leukemia (30). These results link the loss of SLAMF1 expression to the modulation of expression of molecules involved in CLL recirculation to the lymphoid organs, suggesting that this behavior may be responsible, at least partially, for the more aggressive clinical course in SLAMF1 ${ }^{\text {lo }}$ than in SLAMF1 ${ }^{\text {hi }}$ patients. Loss of vimentin in the SLAMF ${ }^{\text {lo }}$ subset, while difficult to interpret at the light of controversial data on the role of this protein in leukemia, suggests that tumor cells undergo a radical reorganization of their cytoskeleton $(58,59)$. A link between SLAMF1 expression and the regulation of cell movement was recently proposed to rely on the modulation of ROS levels, suggesting that different members of the SLAMF family have contrasting properties related to their ability to induce ROS (38). In agreement with those results, we observed downregulation of ROS levels in Mec-1/SLAMF1 ${ }^{\text {sh }}$ cells.

The second finding from gene expression profiling indicates profound modulation of pathways connected with vesicle formation and recirculation. Loss of SLAMF1 affected the expression of a number of molecules that critically regulate this signaling cascade, including VPS34 itself and vimentin. According to published data, beclin1 would serve as a scaffold for the formation of the autophagic complex, while vimentin would be essential in anchoring the vesicles to the cytoskeleton (60).

Intriguingly, cross-linking of SLAMF1 with an agonisic mAb in primary cells and in a representative cell line enhanced the generation of autophagic vesicles and their fusion with the lys- osomes. The finding that autophagy was induced upon SLAMF1 cross-linking with an agonistic monoclonal antibody suggests that the antibody is standing in for a physiological ligand, possibly a second SLAMF1 molecule. If this is true, then it is reasonable to speculate that SLAMF1-SLAMF1 interactions, which occur regularly during normal T-B lymphocyte interactions, would promote autophagy, a mechanism that is increasingly being recognized as important in the regulation of lymphocyte proliferation, energy metabolism, and cell death (61-63). The molecular cascade connecting SLAMF1 to the activation of autophagy relies on the accumulation of ROS, the activation of the MAP kinases, and the phosphorylation of BCL2. This last step causes the dissociation between BCL2 and beclin1 and the consequent assembly of the autophagic macrocomplex, which contains SLAMF1 itself, beclin1, and VPS34. While we cannot exclude the participation of other molecular mechanisms to the formation of the autophagy macrocomplex, the use of specific inhibitors indicates that BCL2 phosphorylation and consequent dissociation from beclin1 is certainly involved.

The third finding of this work is that the expression of SLAMF1 conditions responses to therapeutic agents that act by modulating autophagy. This has been conclusively shown for fludarabine (54) and for a novel class of therapeutic agents that act by causing the degradation of BCL2 (64). The prototype of these drugs is ABT737, which induces the dissociation between BCL2 and beclin1, directly activating autophagy (65). Our experiments indicated that Mec-1/SLAMF1 ${ }^{\text {sh }}$ clones displayed marked resistance to fludarabine- or ABT-737-induced apoptosis. Furthermore, when examining primary cells from patients, it was clear that SLAMF1 $1^{\text {lo }}$ cells were significantly more resistant to fludarabine or ABT-737 than their counterparts, as confirmed by comparing the responses in SLAMF1 ${ }^{\text {hi }}$ and SLAMF1 ${ }^{\text {lo }}$ subclones, obtained from the same CLL patient. Consistent with the hypothesis that the autophagic response is blocked, ROS levels were significantly lower in the Mec-1/SLAMF1 ${ }^{\text {sh }}$ cells than in the controls.

The evidence presented in this paper indicates that SLAMF1 plays as a critical role in CLL homeostasis. Loss of SLAMF1 expression changes the chemotactic responses of CLL cells, likely favoring homing to growth-favorable districts, a process driven by CXCL12. Furthermore, SLAMF1- cells show impairment in the activation of autophagy, which limits their responses to a wide array of therapeutic agents. Restoring SLAMF1 expression in CLL cells would therefore be of therapeutic value for patients with aggressive CLL.

\section{Methods}

Patients and cells. Blood samples were obtained after informed consent. Patient characteristics are reported in Supplemental Table 1. Blood samples of healthy donors (HD) of a comparable age were obtained through the local blood bank. Purified B-lymphocytes were prepared as described (27). Full details are provided in Supplemental Methods. CLL cells were obtained from previously untreated patients or from patients who had not been treated in the previous 6 months. The treatment regimens used are listed in Supplemental Table 1. The Mec-1 cell line was obtained from DSMZ.

Cells were cultured in RPMI-1640 + 10\% FCS and antibiotics (Sigma-Aldrich). 
Antibodies and reagents. A list of antibodies and inhibitors used is provided in Supplemental Methods. Chloroquine diphosphate was from Tocris Bioscience. Protein G-sepharose magnetic beads were from GE Healthcare. Fludarabine and the BH3 mimetic ABT-737 were from Selleckchem.

Generation of SLAMF1-silenced cells. The pGFP-V-RS construct for SLAMF1 shRNA (TG309422), a noneffective (scrambled) shRNA cassette (TR30013) as a negative control and an empty vector (TR3007) were from OriGene. Mec-1 cells were nucleofected with 3 SLAMF1-specific shRNAs (Supplemental Figure 3A) using the Cell Line V Nucleofector Kit (Lonza) and selected by puromycin (SigmaAldrich). SLAMF1- cells were sorted using a FACSAria III cell sorter (BD Biosciences) and cloned by serial dilution. Two clones were established from the cells transfected with shRNA\#1 (5A4 and 5D7) and 1 from the cells transfected with an equimolar mixture of the 3 shRNAs (3F11). Three different clones for Mec-1/Ctrl ${ }^{\text {sh }}$ were used. To generate SLAMF1 shRNA-resistant constructs, 3 synonymous mutations were introduced into the shRNA\#1 targeted region of WT SLAMF1 cDNA clone, purchased from OriGene (pCMV6-Entry construct, RC223343). Mutagenesis was performed using QuikChange II XL Site-Directed Mutagenesis kit (Agilent Technologies). SLAMF1 mutants were verified by Sanger sequencing (Supplemental Figure 5C). Primers used for mutagenesis and sequencing are listed in Supplemental Table 2. The constructs were then transfected into Mec-1/ SLAMF $1^{\text {sh }}$ generated from shRNA\#1, and expression was verified by surface staining with anti-SLAMF1 antibodies, with SLAMF1 mut2 showing the highest percentages of expression (range 15\%-25\%) (Supplemental Figure 5D). Cells transfected with SLAMF1/mut2 were then flow sorted, RNA was extracted, and gene expression was verified by qPCR. Mock construct pCMV6-Entry (OriGene, PS100001) was used as the control.

Gene expression profiling analysis. RNA (300 ng) from Mec-1/ $\mathrm{Ctrl}^{\mathrm{sh}}$ and Mec-1/SLAMF1 ${ }^{\text {sh }}$ clones was amplified and biotinylated using Illumina TotalPrep RNA Amplification Kit. cRNA (750 ng) was hybridized to HumanHT-12 v4 Expression BeadChips using Whole-Genome Expression Direct Hybridisation kit and scanned with iScan System (Illumina). Scanned image analysis and determination of the detection call for each probe set were obtained with the GenomeStudio Gene Expression Module Software (Illumina), applying standard quantile normalization. Expression values were background subtracted and filtered against a $P$ value threshold of 0.01. Unsupervised clustergram analysis of the whole probe set was performed using the clustergram tool of the MATLAB bioinformatics package (http://www.mathworks.it/), using a Pearson correlation coefficient as metric. Lists of differentially expressed genes were constructed on probes (i) that simultaneously passed the $P$ value threshold in all clones examined and (ii) whose minimum fold-change was higher than one in absolute value $(|\log F c|>1)$. Analysis of differentially expressed sequences is described in Supplemental Methods. All microarray data have been deposited in NCBI's Gene Expression Omnibus (GEO GSE69165).

RNA extraction and $q R T-P C R$. RNA was extracted using the RNeasy Plus Mini kit (QIAGEN) and converted to cDNA using the High Capacity cDNA Reverse Transcription kit (Applied Biosystems, Invitrogen).

qRT-PCR was performed using the 7900 HT Fast Real Time PCR system (SDS 2.3 software). A list of primers is provided in Sup- plemental Methods. All reactions were performed in triplicate. The threshold cycle (CT) method was used to calculate expression relative to the endogenous control.

Chemotaxis assay. Chemotaxis assays were performed using the Boyden chamber assay, as described (26). Full details are provided in Supplemental Methods.

Western blot and immunoprecipitation studies. Full details are provided in Supplemental Methods.

Autophagy detection. To detect LC3B-II production, cells were incubated with anti-SLAMF1 functional grade purified (A12) mAb (1.5 $\mu \mathrm{g} / 10^{6}$ cells), in the presence of coated donkey anti-mouse IgG Ab (10 $\mu \mathrm{g} / \mathrm{ml}$ ) or with an irrelevant isotype-matched antibody. Cells were cultured ( 6 hours, $37^{\circ} \mathrm{C}$ ) in normal medium with or without the lysosome inhibitor chloroquine $(15 \mu \mathrm{M})$, were lysed, and were analyzed by Western blot by using the specific antibody anti-LC3B (1:1000).

For confocal microscopy detection of autophagy, treated cells $\left(3 \times 10^{5}\right.$ cells/slide) were centrifuged by cytospin, fixed ( $4 \%$ paraformaldehyde, 10 minutes), permeabilized (0.1\% saponin, 20 minutes), and stained with the indicated antibodies. Nuclei were counterstained with DAPI (Invitrogen). Slides were mounted in SlowFade Gold reagent (Invitrogen) and analyzed using a TCS SP5 laser scanning confocal microscope with 4 lasers (Leica Microsystems); images were acquired with LAS AF Version Lite 2.4 software (Leica Microsystems) and processed with Adobe Photoshop CS6 software.

For electron microscopy detection of autophagy, purified CLL cells $\left(6 \times 10^{6}\right)$ were fixed $(2.5 \%$ glutaraldheyde in $0.1 \mathrm{M}$ cacodilate buffer ( $\mathrm{pH}$ 7.2), 2 hours, room temperature and then overnight, $4^{\circ} \mathrm{C}$ ), rinsed, and dehydrated in an ascending series of ethanol solutions to $100 \%$. Cells were then infiltrated in Epon-Araldite resin, which polymerized (24 hours, $60^{\circ} \mathrm{C}$ ) (66). Embedded samples were processed for ultramicrotomy: semithin sections $(0.5 \mu \mathrm{m})$ were stained with $1 \%$ toluidine blue, and ultrathin $(70 \mathrm{~nm})$ sections were counterstained with uranyl acetate and lead citrate. Samples were examined on a Philips CM10 transmission electron microscope.

ROS production. Intracellular ROS production was detected by flow cytometry using the redox sensitive dyeCM- $\mathrm{H}_{2}$ DCFDA $(1 \mu \mathrm{M}, 20$ minutes, $37^{\circ} \mathrm{C}$ ) or the CellROX Deep Red Reagent fluorogenic probe $\left(1 \mu \mathrm{M}, 30\right.$ minutes, $\left.37^{\circ} \mathrm{C}\right)$ following manufacturer instructions. All probes were from Molecular Probes (Invitrogen).

For NOX2 inhibition studies, cells were transfected with $60 \mathrm{pmol} /$ sample of gp91-phox siRNA, p40-phox siRNA, or negative control siRNA (all from Santa Cruz Biotechnology Inc.).

Statistics. The cumulative probability of TFS and OS were estimated by recursive partitioning analysis. TFS was defined as the time elapsed from date of diagnosis to date of first treatment, and OS was defined as the time from first diagnosis to death. The analysis was performed with the Statistical Package for the Social Sciences (spss) software v.16.0 (SPSS Inc.).

Cumulative data of functional studies are shown as mean values with standard deviation and statistical significance determined using Mann-Whitney $U$ test (unpaired data) or Wilcoxon signed rank test (paired data), as appropriate. Matched groups (3 or more) were compared using Friedman's test with Dunn's multiple-comparisons test. Correlation between continuous variables was assessed using Pearson's coefficient.

Data are reported as box plots, where the top and bottom of the rectangle define the third and first quartile, respectively, and the whis- 
kers define the maximum and minimum values. The line within the rectangle represents the median value. In some instances, data are reported as bar graphs, where the height of the bar represents the mean value, while the error bars are the standard deviation.

Statistical analyses were performed using Prism software Version 5.0 (GraphPad Software), and the statistical significance was defined as $P<0.05$.

Study approval. Blood samples were obtained after informed consent, in accordance with Institutional Guidelines and the Declaration of Helsinki. The study was approved by the Hugef Ethics Committee.

\section{Author contributions}

$\mathrm{CB}, \mathrm{RB}, \mathrm{SS}, \mathrm{TV}, \mathrm{VA}, \mathrm{DB}$, and EM designed and conducted experiments and analyzed data. AP and RP analyzed data. MC, GDA, and RRF provided patient samples. DR and GG provided patient samples and analyzed data. CT discussed and analyzed data. SD designed experiments and wrote the manuscript.

\section{Acknowledgments}

Thanks are given to Leandro Cerchietti (Weill Cornell Medical College, New York, New York, USA) for helpful discussions. We also thank Maria Lamusta and Katiuscia Gizzi for technical support. This work was supported by grants from the Italian Ministries of Education, University and Research (Futuro in Ricerca 2012, RBFR12D1CB), the Italian Ministry of Health (Bando Giovani Ricercatori 2008, GR-2008-1138053, GR-2010-2317594, and GR-201102349282), the Associazione Italiana per la Ricerca sul Cancro AIRC (IG 12754 and Special Program Molecular Clinical Oncology 5 $\times 1000$ No. 10007), and the Fondazione Cariplo, grant 2012-0689. V. Audrito is supported by a triennial FIRC/AIRC fellowship (15047).

Address correspondence to: Silvia Deaglio, Department of Medical Sciences, University of Turin School of Medicine and HuGeF, via Nizza, 52, 10126 Torino, Italy. Phone:39.011. 670.9535; E-mail: silvia.deaglio@unito.it.
1. Chiorazzi N, Rai KR, Ferrarini M. Chronic lymphocytic leukemia. $N$ Engl J Med. 2005;352(8):804-815.

2. Gaidano G, Foa R, Dalla-Favera R. Molecular pathogenesis of chronic lymphocytic leukemia. J Clin Invest. 2012;122(10):3432-3438.

3. Burger JA, Chiorazzi N. B cell receptor signaling in chronic lymphocytic leukemia. Trends Immunol. 2013;34(12):592-601.

4. Dal Bo M, et al. Microenvironmental interactions in chronic lymphocytic leukemia: the master role of CD49d. Semin Hematol. 2014;51(3):168-176.

5. Malavasi F, Deaglio S, Damle R, Cutrona G, Ferrarini M, Chiorazzi N. CD38 and chronic lymphocytic leukemia: a decade later. Blood. 2011;118(13):3470-3478.

6. Dohner H, et al. Genomic aberrations and survival in chronic lymphocytic leukemia. $N$ Engl J Med. 2000;343(26):1910-1916.

7. Mertens D, et al. Chronic lymphocytic leukemia and 13q14: miRs and more. Leuk Lymphoma. 2009;50(3):502-505.

8. Cimmino A, et al. miR-15 and miR-16 induce apoptosis by targeting BCL2. Proc Natl Acad Sci US A. 2005;102(39):13944-13949.

9. Ng SY, Davids MS. Selective Bcl-2 inhibition to treat chronic lymphocytic leukemia and nonHodgkin lymphoma. Clin Adv Hematol Oncol. 2014;12(4):224-229.

10. Klionsky DJ. Autophagy: from phenomenology to molecular understanding in less than a decade. Nat Rev Mol Cell Biol. 2007;8(11):931-937.

11. Levine B, Kroemer G. Autophagy in the pathogenesis of disease. Cell. 2008;132(1):27-42.

12. Strohecker AM, White E. Targeting mitochondrial metabolism by inhibiting autophagy in BRAFdriven cancers. Cancer Discov. 2014;4(7):766-772.

13. Kang R, Zeh HJ, Lotze MT, Tang D. The Beclin 1 network regulates autophagy and apoptosis. Cell Death Differ. 2011;18(4):571-580.

14. White E. Deconvoluting the context-dependent role for autophagy in cancer. Nat Rev Cancer. 2012;12(6):401-410.

15. Galluzzi L, et al. Autophagy in malignant transformation and cancer progression. $E M B O J$. 2015;34(7):856-880.
16. Huang JJ, Zhu YJ, Lin TY, Jiang WQ, Huang HQ, Li ZM. Beclin 1 expression predicts favorable clinical outcome in patients with diffuse large B-cell lymphoma treated with R-CHOP. Hum Pathol. 2011;42(10):1459-1466.

17. Calpe S, et al. The SLAM and SAP gene families control innate and adaptive immune responses. Adv Immunol. 2008;97:177-250.

18. Veillette A. Immune regulation by SLAM family receptors and SAP-related adaptors. Nat Rev Immunol. 2006;6(1):56-66.

19. Engel P, Eck MJ, Terhorst C. The SAP and SLAM families in immune responses and $\mathrm{X}$-linked lymphoproliferative disease. Nat Rev Immunol. 2003;3(10):813-821.

20. Berger SB, et al. SLAM is a microbial sensor that regulates bacterial phagosome functions in macrophages. Nat Immunol. 2010;11(10):920-927.

21. Seifert M, et al. Cellular origin and pathophysiology of chronic lymphocytic leukemia. JExp Med. 2012;209(12):2183-2198.

22. Mittal AK, et al. Molecular basis of aggressive disease in chronic lymphocytic leukemia patients with 11q deletion and trisomy 12 chromosomal abnormalities. Int JMol Med. 2007;20(4):461-469.

23. Zucchetto A, et al. Cluster analysis of immunophenotypic data: the example of chronic lymphocytic leukemia. Immunol Lett. 2011;134(2):137-144.

24. Schweighofer CD, et al. A two-gene signature, SKI and SLAMF1, predicts time-to-treatment in previously untreated patients with chronic lymphocytic leukemia. PLoS One. 2011;6(12):e28277.

25. Isomaki $\mathrm{P}$, et al. Increased expression of signaling lymphocytic activation molecule in patients with rheumatoid arthritis and its role in the regulation of cytokine production in rheumatoid synovium. Jimmunol. 1997;159(6):2986-2993.

26. Deaglio S, et al. CD38 and ZAP-70 are functionally linked and mark CLL cells with high migratory potential. Blood. 2007;110(12):4012-4021.

27. Vaisitti T, et al. CD38 increases CXCL12-mediated signals and homing of chronic lymphocytic leukemia cells. Leukemia. 2010;24(5):958-969.

28. Buggins AG, et al. Evidence for a macromolecular complex in poor prognosis CLL that contains CD38, CD49d, CD44 and MMP-9. Br J Haema- tol. 2011;154(2):216-222.

29. Deaglio S, Vaisitti T, Zucchetto A, Gattei V, Malavasi F. CD38 as a molecular compass guiding topographical decisions of chronic lymphocytic leukemia cells. Semin Cancer Biol. 2010;20(6):416-423.

30. Ocana E, et al. The prognostic role of CXCR3 expression by chronic lymphocytic leukemia $\mathrm{B}$ cells. Haematologica. 2007;92(3):349-356.

31. Zucchetto A, et al. A scoring system based on the expression of six surface molecules allows the identification of three prognostic risk groups in B-cell chronic lymphocytic leukemia. J Cell Physiol. 2006;207(2):354-363.

32. Wang RC, et al. Akt-mediated regulation of autophagy and tumorigenesis through Beclin 1 phosphorylation. Science. 2012;338(6109):956-959.

33. Funderburk SF, Wang QJ, Yue Z. The Beclin 1-VPS34 complex - at the crossroads of autophagy and beyond. Trends Cell Biol. 2010;20(6):355-362.

34. Mizushima N, Yoshimori T, Levine B. Methods in mammalian autophagy research. Cell. 2010;140(3):313-326.

35. Mizushima N, Yoshimori T. How to interpret LC3 immunoblotting. Autophagy. 2007;3(6):542-545.

36. Scherz-Shouval R, Elazar Z. ROS, mitochondria and the regulation of autophagy. Trends Cell Biol. 2007;17(9):422-427.

37. Liu P, et al. Dysregulation of TNF $\alpha$-induced necroptotic signaling in chronic lymphocytic leukemia: suppression of CYLD gene by LEF1. Leukemia. 2012;26(6):1293-1300.

38. Wang G, et al. Migration of myeloid cells during inflammation is differentially regulated by the cell surface receptors Slamf1 and Slamf8. PLoS One. 2015;10(3):e0121968.

39. Ogata M, et al. Autophagy is activated for cell survival after endoplasmic reticulum stress. Mol Cell Biol. 2006;26(24):9220-9231.

40. Corcelle E, et al. Control of the autophagy maturation step by the MAPK ERK and p38: lessons from environmental carcinogens. Autophagy. 2007;3(1):57-59.

41. Matsunaga K, et al. Two Beclin 1-binding proteins, Atg14L and Rubicon, reciprocally regulate autophagy at different stages. Nat Cell Biol. 
2009;11(4):385-396.

42. Zhong Y, et al. Distinct regulation of autophagic activity by Atg14L and Rubicon associated with Beclin 1-phosphatidylinositol-3-kinase complex. Nat Cell Biol. 2009;11(4):468-476.

43. Pattingre S, et al. Bcl-2 antiapoptotic proteins inhibit Beclin 1-dependent autophagy. Cell. 2005;122(6):927-939.

44. Backer JM. The regulation and function of Class III PI3Ks: novel roles for Vps34. Biochem J. 2008;410(1):1-17.

45. Wei Y, Pattingre S, Sinha S, Bassik M, Levine B. JNK1-mediated phosphorylation of Bcl-2 regulates starvation-induced autophagy. Mol Cell. 2008;30(6):678-688.

46. Wei Y, Sinha S, Levine B. Dual role of JNK1mediated phosphorylation of Bcl-2 in autophagy and apoptosis regulation. Autophagy. 2008;4(7):949-951.

47. Pietrocola F, et al. Regulation of autophagy by stress-responsive transcription factors. Semin Cancer Biol. 2013;23(5):310-322.

48. Kroemer G, Marino G, Levine B. Autophagy and the integrated stress response. Mol Cell. 2010;40(2):280-293.

49. Ricci F, Tedeschi A, Morra E, Montillo M. Fludarabine in the treatment of chronic lymphocytic leukemia: a review. Ther Clin Risk Manag. 2009;5(1):187-207.

50. Pedro JM, et al. BAX and BAK1 are dispensable for ABT-737-induced dissociation of the BCL2-
BECN1 complex and autophagy. Autophagy. 2015;11(3):452-459.

51. Notte A, Leclere L, Michiels C. Autophagy as a mediator of chemotherapy-induced cell death in cancer. Biochem Pharmacol. 2011;82(5):427-434.

52. Ekiz HA, Can G, Baran Y. Role of autophagy in the progression and suppression of leukemias. Crit Rev Oncol Hematol. 2012;81(3):275-285.

53. Nencioni A, et al. Autophagy in blood cancers: biological role and therapeutic implications. Haematologica. 2013;98(9):1335-1343.

54. Mahoney E, et al. ER stress and autophagy: new discoveries in the mechanism of action and drug resistance of the cyclin-dependent kinase inhibitor flavopiridol. Blood. 2012;120(6):1262-1273.

55. MacCallum SF, et al. Dysregulation of autophagy in chronic lymphocytic leukemia with the small-molecule Sirtuin inhibitor Tenovin-6. Sci Rep. 2013;3:1275.

56. Mahoney E, Byrd JC, Johnson AJ. Autophagy and ER stress play an essential role in the mechanism of action and drug resistance of the cyclindependent kinase inhibitor flavopiridol. Autophagy. 2013;9(3):434-435.

57. Vaisitti $T$, et al. The enzymatic activities of CD38 enhance CLL growth and trafficking: implications for therapeutic targeting. Leukemia. 2015;29(2):356-368.

58. Satelli A, Li S. Vimentin in cancer and its potential as a molecular target for cancer therapy. Cell Mol Life Sci. 2011;68(18):3033-3046.
59. Nowakowski GS, et al. Percentage of smudge cells on routine blood smear predicts survival in chronic lymphocytic leukemia. J Clin Oncol. 2009;27(11):1844-1849.

60. Ghavami S, et al. Autophagy is a regulator of TGF- $\beta 1$-induced fibrogenesis in primary human atrial myofibroblasts. Cell Death Dis. 2015;6:e1696.

61. Valdor R, et al. Chaperone-mediated autophagy regulates $\mathrm{T}$ cell responses through targeted degradation of negative regulators of $\mathrm{T}$ cell activation. Nat Immunol. 2014;15(11):1046-1054.

62. Pua HH, Dzhagalov I, Chuck M, Mizushima N, He YW. A critical role for the autophagy gene Atg5 in T cell survival and proliferation. J Exp Med. 2007;204(1):25-31.

63. Hubbard VM, Valdor R, Patel B, Singh R, Cuervo AM, Macian F. Macroautophagy regulates energy metabolism during effector $\mathrm{T}$ cell activation. JImmunol. 2010;185(12):7349-7357.

64. Oltersdorf T, et al. An inhibitor of Bcl-2 family proteins induces regression of solid tumours. Nature. 2005;435(7042):677-681.

65. Del Gaizo Moore V, Brown JR, Certo M, Love TM, Novina CD, Letai A. Chronic lymphocytic leukemia requires BCL2 to sequester prodeath BIM, explaining sensitivity to BCL2 antagonist ABT-737. JClin Invest. 2007;117(1):112-121.

66. Reynolds ES. The use of lead citrate at high $\mathrm{pH}$ as an electron-opaque stain in electron microscopy. JCell Biol. 1963;17:208-212. 